المجلد 31 - عدد 4 - ديسمبر 2020، ص.ص.371 -388

\title{
المكانة المستقبلية للمحروقات في الاقتصاد العالمي مع الإشارة إلى حالة الجزائر
}

The future status of hydrocarbons in the global economy with reference to the case of Algeria

تاريخ الاستلام : 2019/07/16 ؛ تاريخ القبول : 2020/10/20

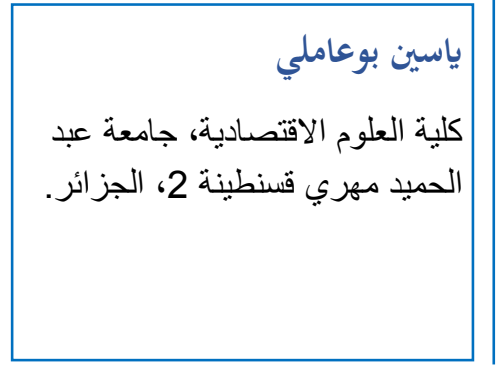

\section{Abstract}

When oil was first discovered in 1859 , it had few uses. As time progressed, modern economic activity has become increasingly dependent on it. This dependence has become a major area of concern following successive oil crises, and has led scientific community, especially geologists and economists to question the peak-Oil. The aim of this article is, on the one hand, the analysis of the arguments of the "Peakists" as well as those of their opponents, and on the other hand, the analysis of the factors that may determine the future place of hydrocarbons, both in the world economy and the Algerian economy. The article concludes that this place will also be affected by several non-geological factors and not only related to current estimates of the reserves of these resources.

Keywords: fossil fuels; peak-oil ; unconventional hydrocarbons; alternative energy.

\section{Résumé}

À sa découverte pour la première fois en 1859 , le pétrole n'avait que peu d'usages. Cependant, au fil du temps, l'activité économique en est devenue de plus en plus dépendante. Cette dépendance est devenue un sujet de préoccupations majeur suite aux crises pétrolières successives et a conduit la communauté scientifique, notamment les géologues et les économistes, à s'interroger sur le Peak-Oil. L'objectif de cet article est, d'une part, l'analyse des arguments des "Peakists" ainsi que ceux de leurs opposants, et d'autres parts, l'analyse des facteurs qui pourront déterminer la place future des hydrocarbures, aussi bien dans l'économie mondiale que l'économie algérienne. L'article conclut que cette place sera également affectée par plusieurs facteurs non géologiques et non seulement liée aux estimations actuelles des réserves de ces ressources.

Mots clés : combustibles fossiles; pic pétrolier ; hydrocarbures non conventionnelles; énergie alternative.

* Corresponding author, e-mail: yassine.bouamli@univ-constantine2.dz 


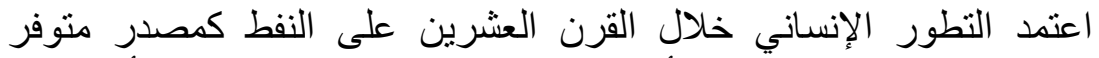

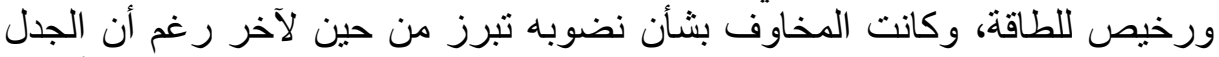

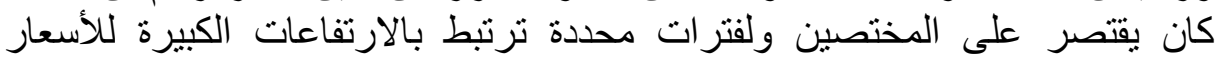

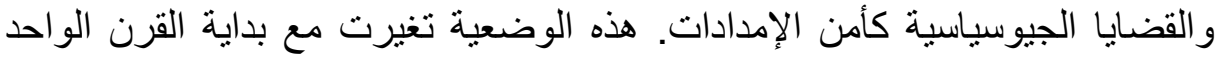

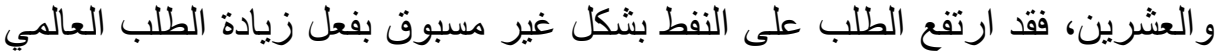

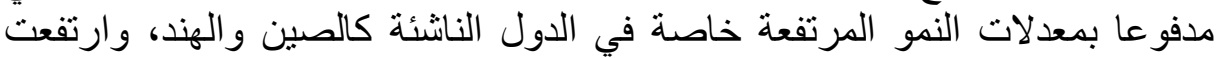

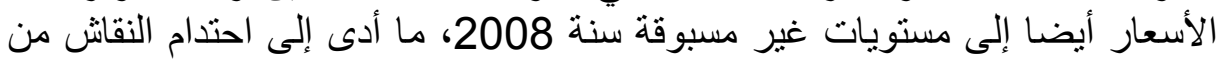

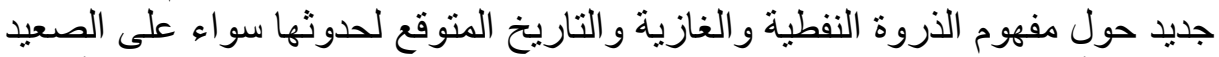

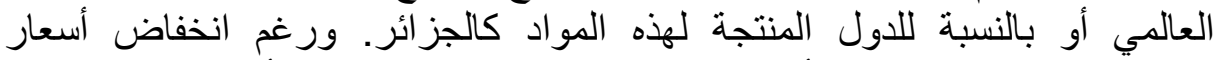

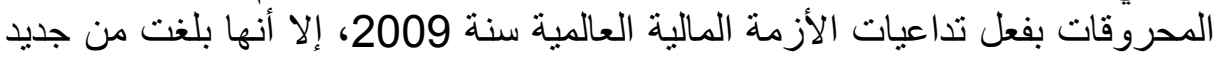

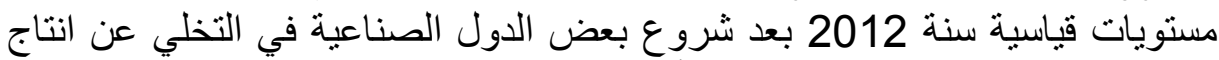

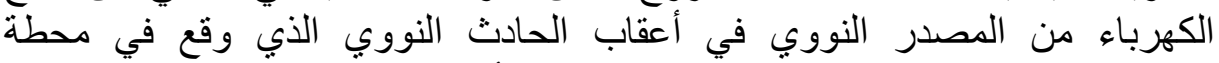

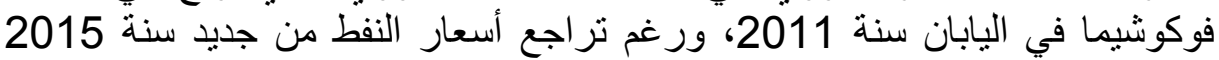

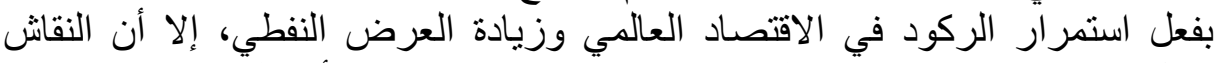

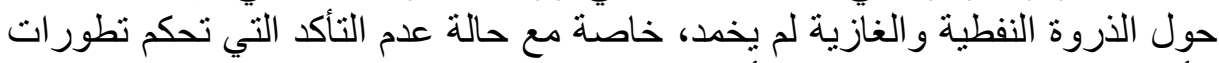

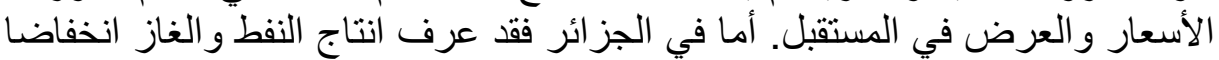

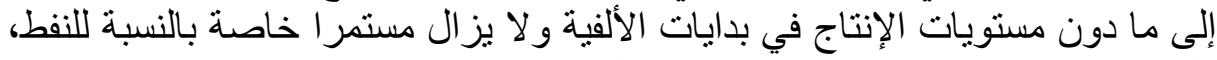

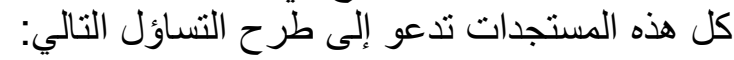

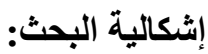

ما هي المكانة المستقبلية للمحروقات في الاقتصاد العالمي والجزائري في

ظل التطور ات العالمية الر اهنة؟ المكات

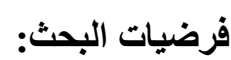

لمعالجة موضو ع البحث تم صياغة الفرضيات التالية:

- سوف تكون هنالك ذروة لإنتاج المحروقات في المستقبل، إلا ان التنبؤ بتاريخها ومنحناها البياني يبقى أمرا غير ممكن سواء لاءنتاج التى الصعيد العالمي أو المحلي.

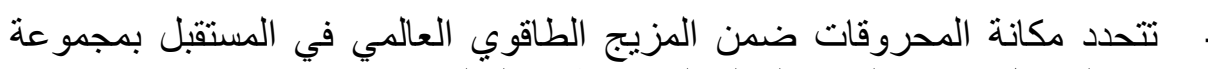
من العو امل تتعدى الحجم الحالي للاحتياطات العالمية.

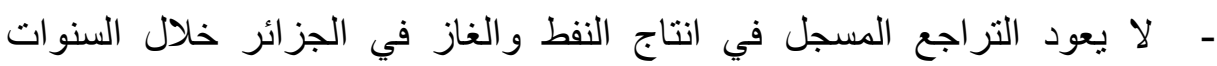

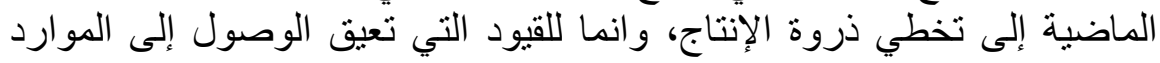

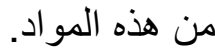

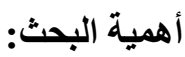

تبرز أهمية البحث في احتلال المحروقات لمكانة هامة ضمن المزيج الطاقوي

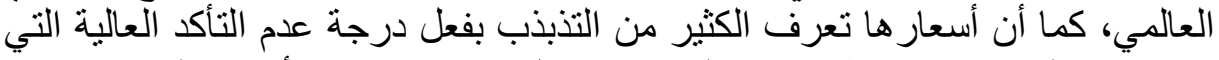
تحكم مستقبلها، وهو ما يطر ح هذه القضية في كل مرة ترتفع فيها أسعار المحروقات.

وسوف نحاول الإجابة على التساؤلات السابقة من خلال العناصر التالية: I الأوضاع الحالية للطاقة على الصعيد العالمي: I قبل التطرق لأوضاع الطاقة على الصعيد العالمي يجب أولا التفريق بين الطاقة 
الأولية و الطاقة النهائية، فالطاقة الأولية هي كل المنتجات الطاقوية التي لم تخضع لأي الي التي

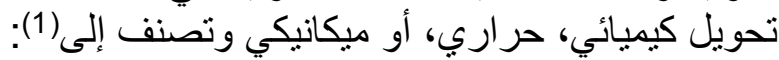

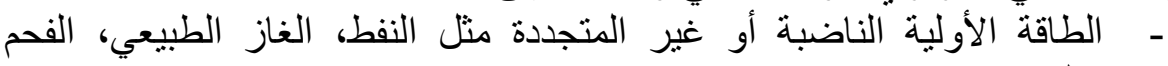

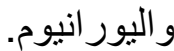

- الطاقة الأولية المتجدة مثل الطاقة المائية، الطاقة الهوائية، الطاقة الثمسية،

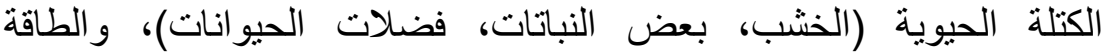

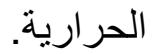
وبعد أن تجرى على هذه المصادر عمليات التحويل والنقل يتم استهلاكها على شكل

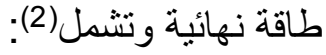
- الطاقة المستعملة من طرف المات المستهلكين النهائيين في قطاعات الصناعة،

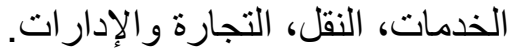

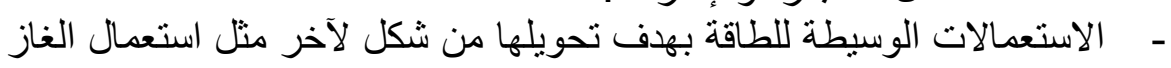

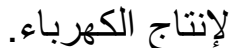
ـ ـ الإنتهلاك الذاتي للموردين بهدف تزوبيد السوق بالطاقة مثل ضخ الغاز لاستخر اج بعض أنواع النفط.

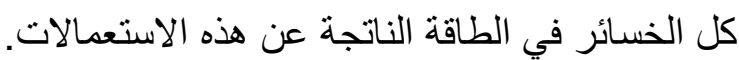

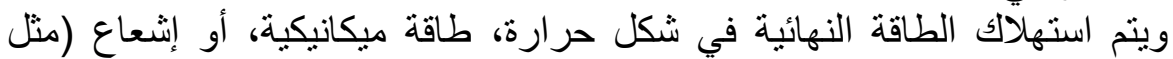

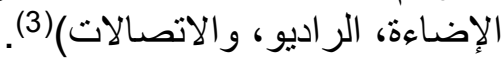
حسب المجلة الإحصائية للطاقة العالمية لبريتش بيتروليوم لسنة 2018، بلغ العادئ

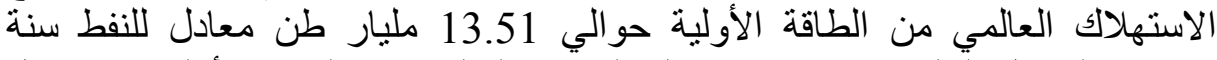

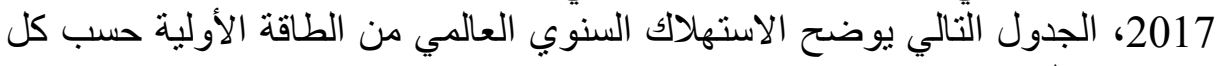
مصدر خلال سنتي 1990 و 2017 التئي

الثكل رقم -1-الاستهلاك العالمي من الطاقة الأولية حسب مصادرها في سنتي 1990

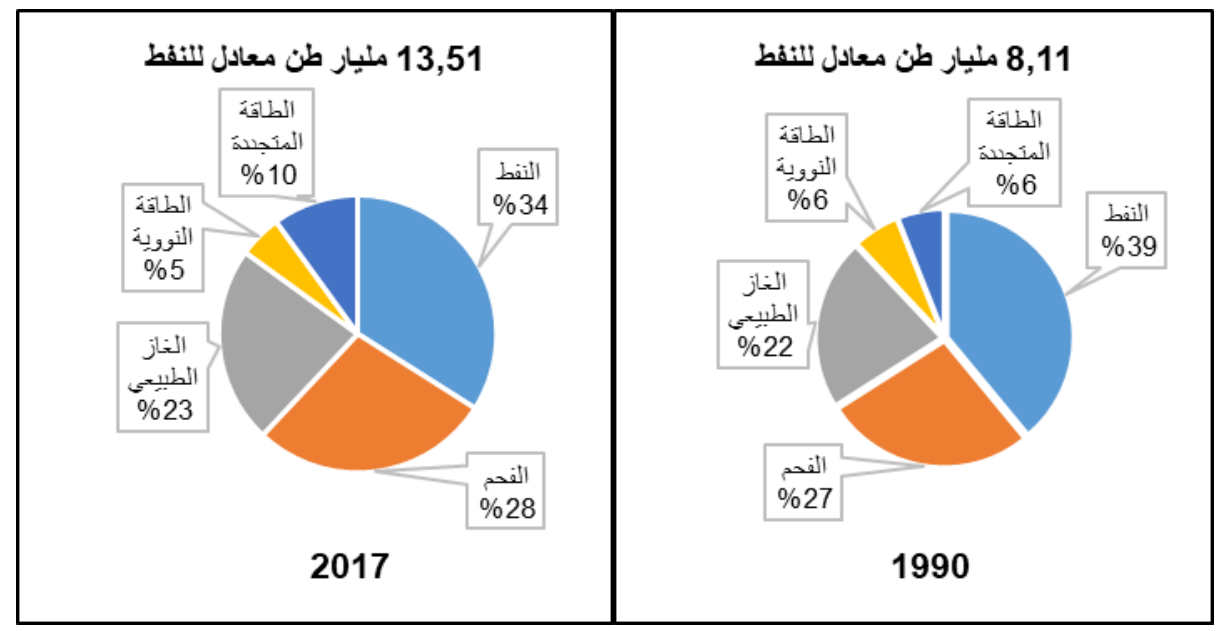

المصدر: تم إعداده اعتمادا على:

British petroleum, BP statistical review of world energy, June 2018.

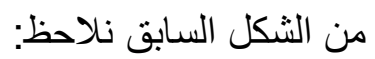

- من الزيادة الكبيرة في الاستهلاك السنوي من الطاقة الأولية بين سنتي 1990 و2017 حيث بلغت حو الي 66\%، وقد عرفت هذه الفترة نموا غير مسبوق الأني في سني 
الطلب العالمي على الطاقة خاصة بفعل النمو السريع في الاقتصاديات

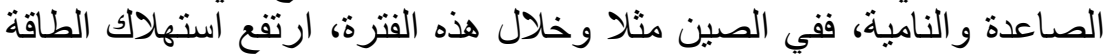

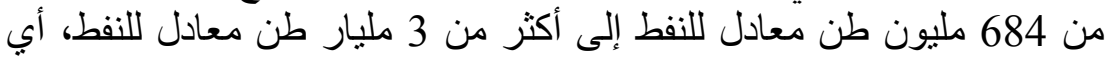

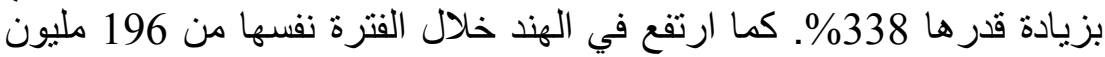

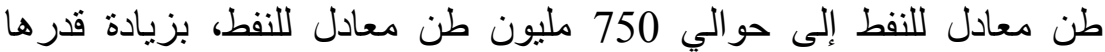
$\% 282$

شكلت المحروقات 88\% من الطلب العالمي على الطاقة سنة 1990 و 858\%

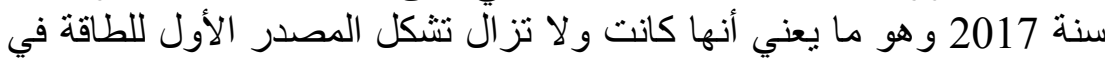

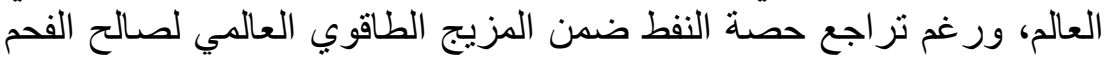

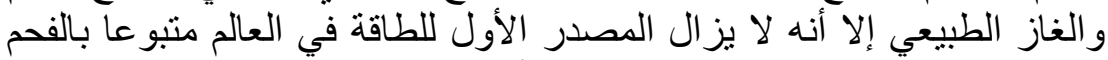

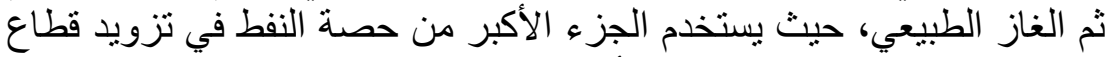

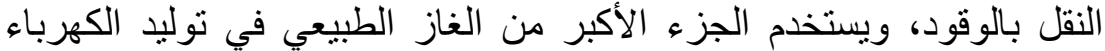

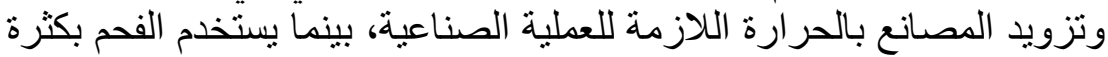

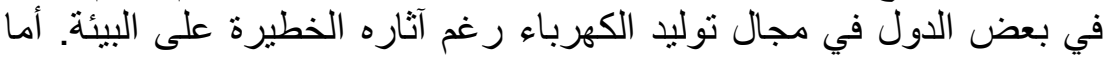

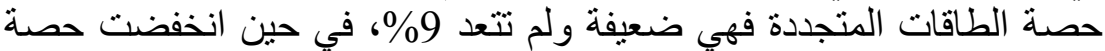

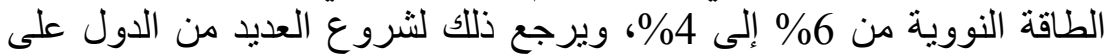

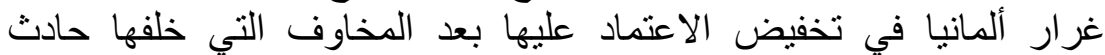

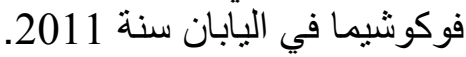
هذا الطلب على مختلف مصادر الطاقة الأولية مرشح للزيانة النادة بشكل كبير في

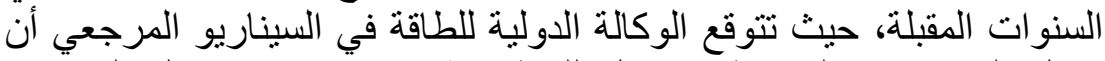

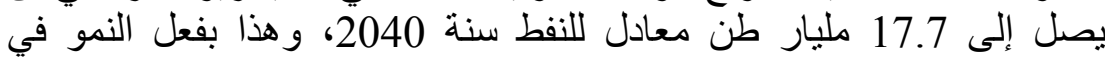

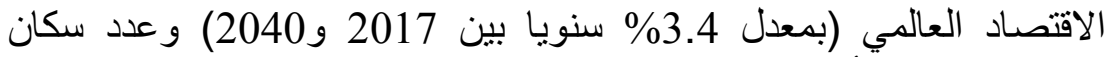
العالم الذي يتوقع أن يصل إلى 9.2 مليار نسمة سنة 2040 (4).

إن النسب الموضحة في الثكل السابق تعكس بنية جهاز الإنتاج والاستهلاك

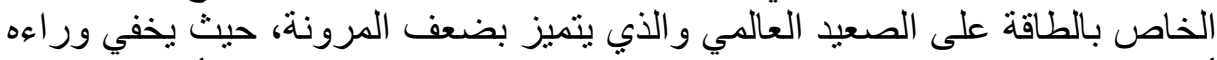

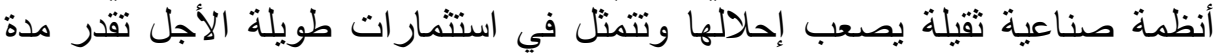

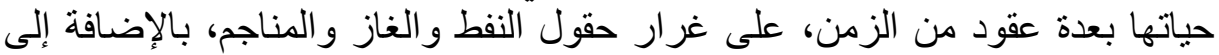

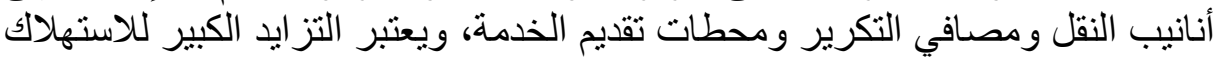

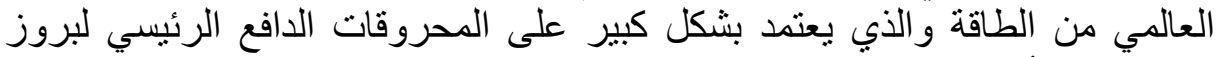

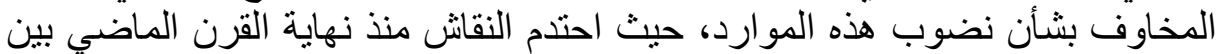

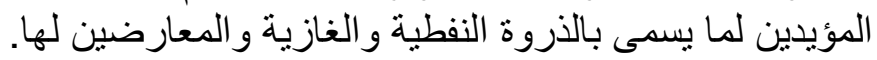

نظرية الذروة النفطية والغازية بين المؤيدين والمعارضين: -II

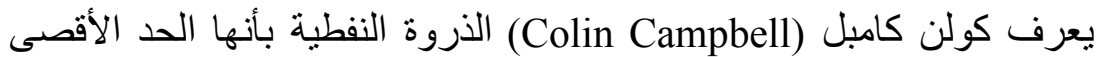

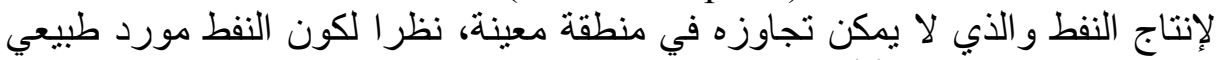

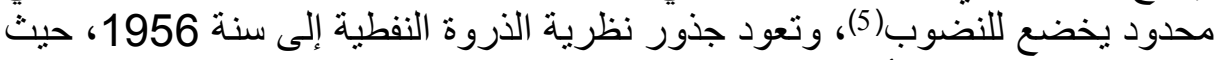

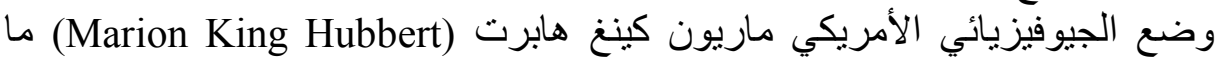

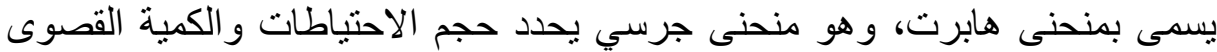

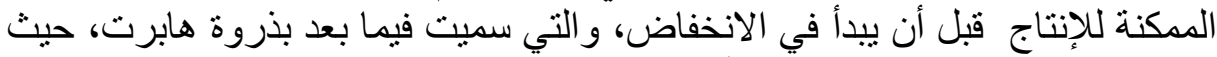

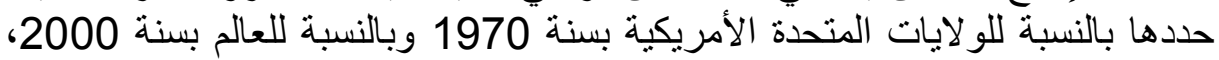
ومع ارتفاع الطلب العالمي على النفط منذ التسعينات نشرت التهنة مجلة العلوم الأمريكية سنة 1988مقالا للجيولوجيين الثهيرين، الفنلندي كولن النين (American Scientific)

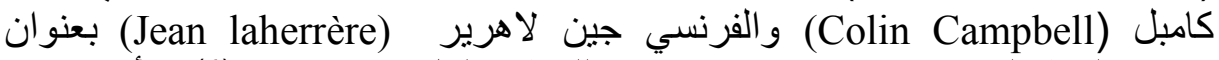
"نهاية النفط الرخيص" نوقع نهاية وشيكة للنفط بحلول سنة 2010(6)، وانيل وأعاد زخم 
النقاش حول مستقبل إنتاج النفط، وفي سنة 2000 قام كامبل و لا هرير بتأسيس جمعية (Association for the Study of Peak Oil and دراسة الذروة النفطية والغازية النازية (Gas) ويمكن تلخيص آر اء أنصار ومعارضي نظة نظية الذروة النفطية فيما يلي:

ألرأي المؤيد (الاتجاه المتثائم):-1-II

معظم أنصار هذا الرأي من الجيولوجيين، وتعتبر جمعية دراسة الذبروة الذباه

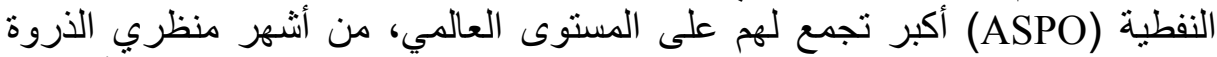

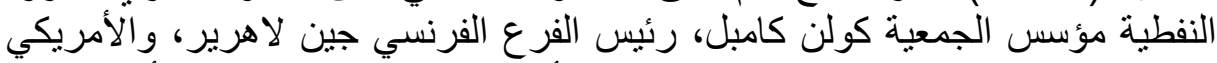

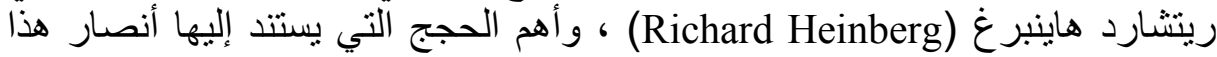

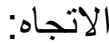

- أهم الاكتشافات النفطية تمت قبل سنوات الثمانينات من القرن الماضي، أما

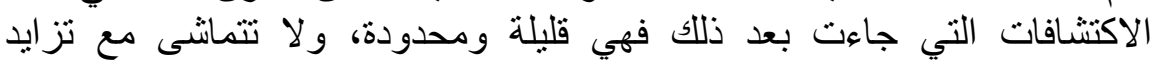
الاستهلاك العالمي من الطاقة.

- ل ل يشكل النفط غير التقليدي سوى جزء ضئيل من الاستهلاك الحالي، ويتطلب

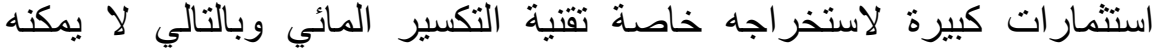
تعوض تناقص إنتاج النفط الخام (7).

- الاحتياطات المثبتة المعلن عنها ليست صحيحة بالنسبة لجميع الدول، وتميز

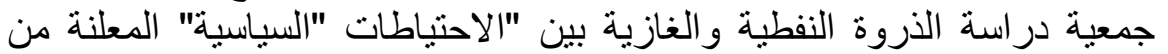

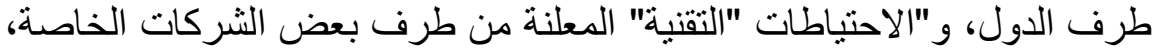
إلا أنها مرتفعة التكاليف و غير مناحة لعموم الباحثين (8).

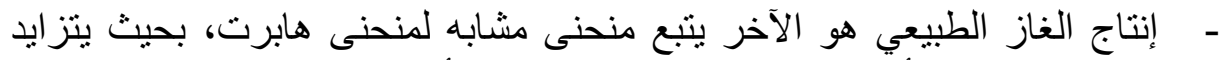

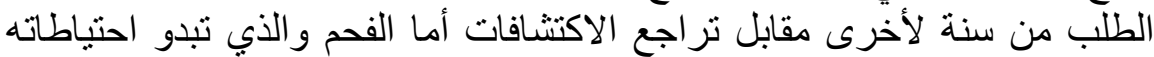

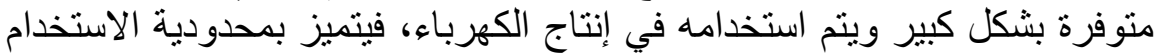
وضعف المردود الطاقوي بالإضافة إلى الجدل الكبير الذي يثيره بئيز بسبب تلويث

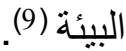

الثكل التالي يوضح منحنى التوقعات المستقبلية لإنتاج النفط في العالم حسب

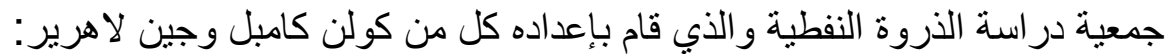

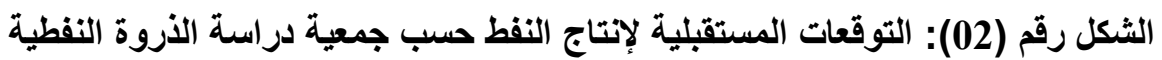

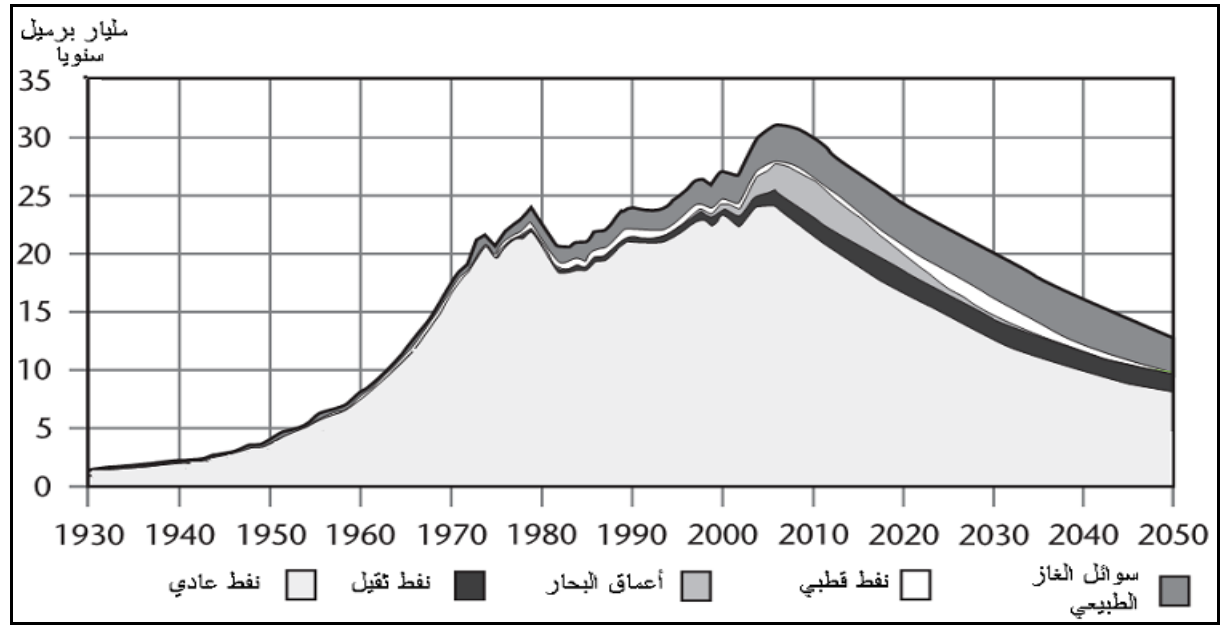

Source: Richard Heinberg, The Party's over, P102. 
من الثكل نجد أن إنتاج النفط التقليدي لن يتجاوز السقف المسجل في حدود سنة

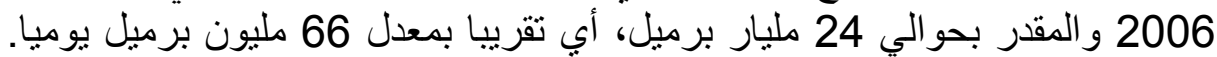

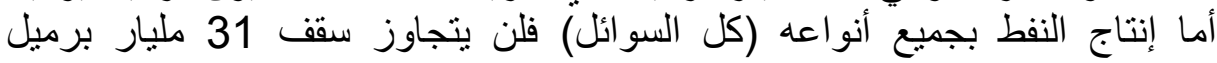

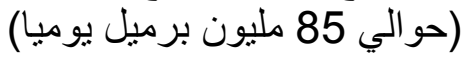
بالرجوع إلى المجلة الإحصائية للطاقة العالمية لبريتش بيتروليوم لبنائة 2018

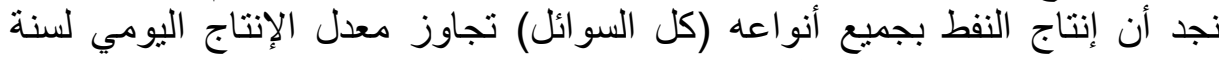
2006، كما يوضحه الثكل التالي:

الشكل رقم (03): تطور الإنتاج العالمي من النفط (كل السوائل) منذ سنة 2000

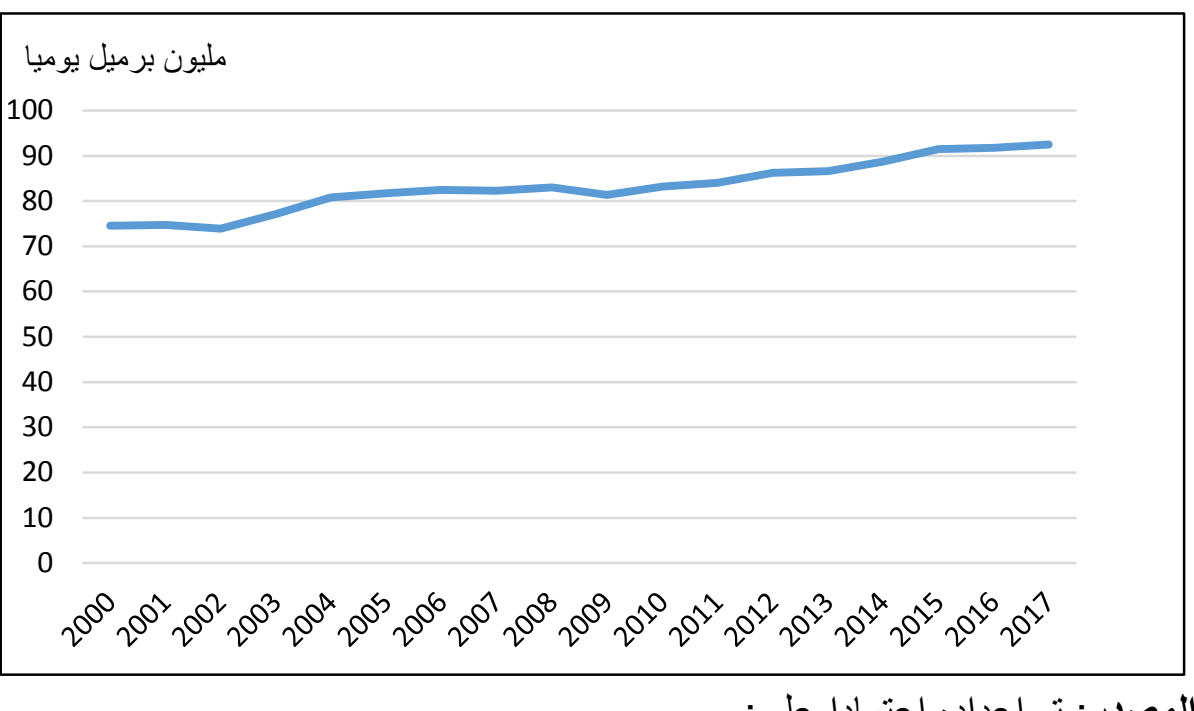

المصدر: تم إعداده اعتمادا على:

British petroleum, BP statistical review of world energy, June 2018.

نلاحظ من الثكل حدوث انخفاض طفيف في الإنتاج العالمي للنفط بين سنتي

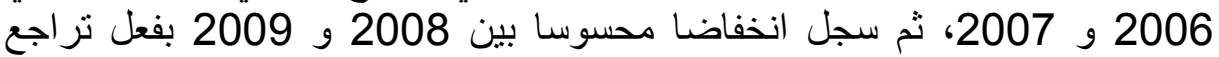
الطلب العالمي الذي سبيته الازمة الاقتصادية العالمية خلال هذه الفترة الفان، ليعود الاتجاه

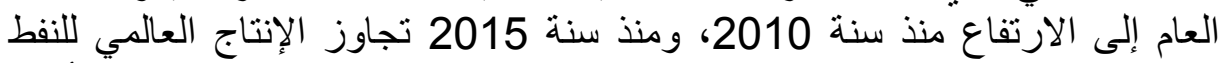
سقف 91 مليون برميل يوميا بسبب زيادة العرض من طرف روفي روسيا ودول الأوبك العالك

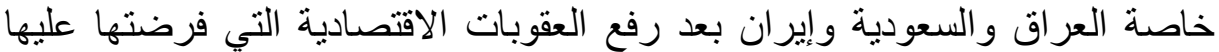

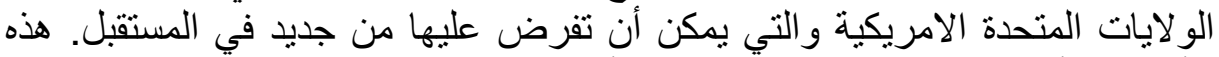

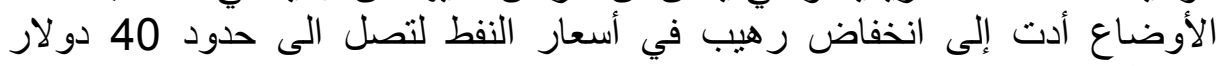

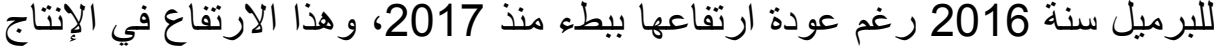

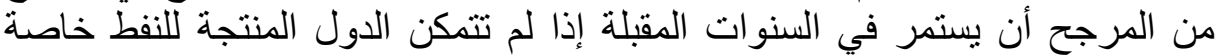

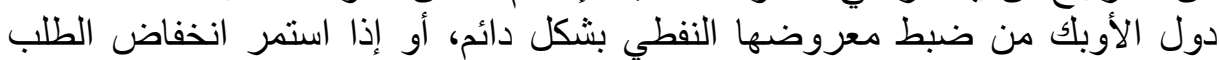

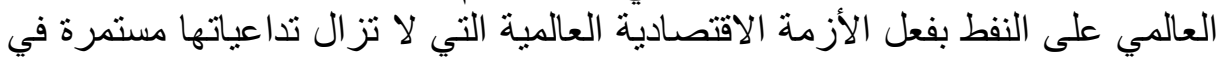
الكثير من الدول المستهلكة للنفط، كل هذه العوامل قد تدفع بالذروة النفطية لسنوات

2-II

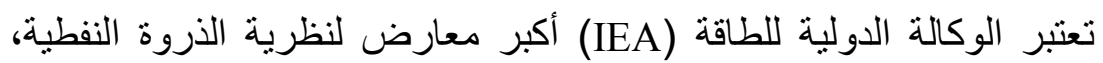
بالإضافة إلى دائرة معلومات الطاقة (EIA) في الولايات المتحدة الأمريكية ومجلس النس 
الطاقة العالمي (WEC) و الثركات النفطية، وأهم الحجج التي يستند إليها أنصار هذا

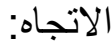

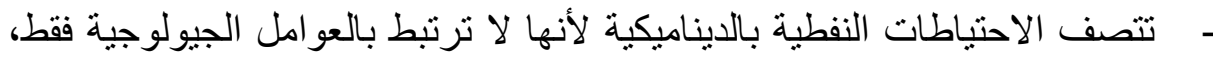

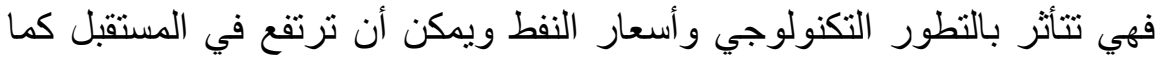
ارتفعت في السنوات الماضية كما يبينه الجدول النالي:

الجدول رقم (01): الاحتياطات المثبتة والإنتاج السنوي من النفط في العالم خلال

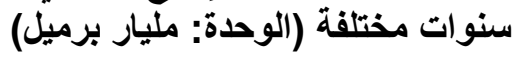

\begin{tabular}{|c|c|c|c|}
\hline 2017 & 2004 & 1994 & 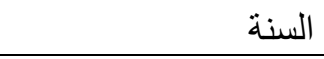 \\
\hline 1697 & 1366 & 1118 & الاحتباطات المثبتة (R) \\
\hline 34 & 30 & 24 & الإنتاج السنوي (P) \\
\hline 50 & 46 & 47 & R/P (بالسنو ات) \\
\hline
\end{tabular}

British petroleum, BP statistical review of world energy, June 2015 and June 2018.

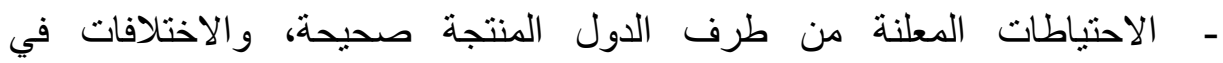
الإحصائيات العالمية للطاقة تخص حنى ما يسمى بالإحصائيات التقنية.

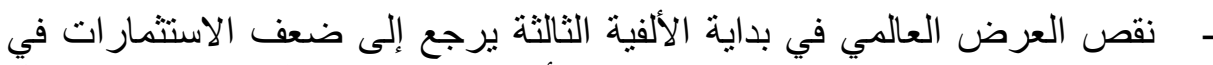

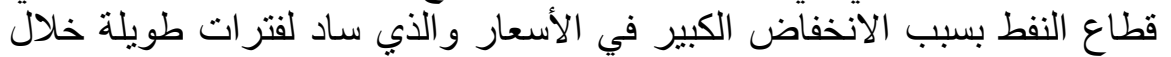

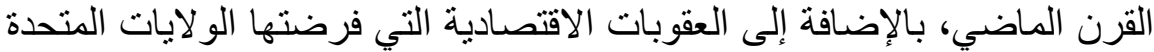

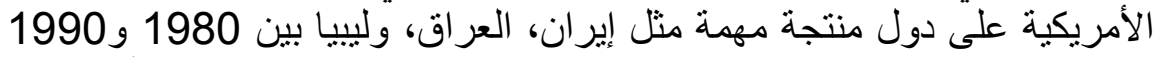

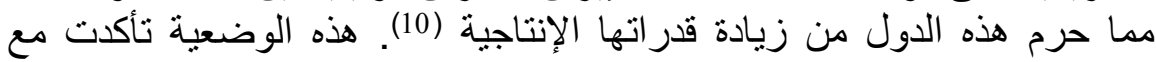

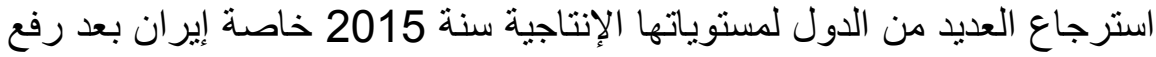

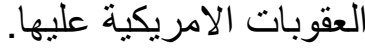

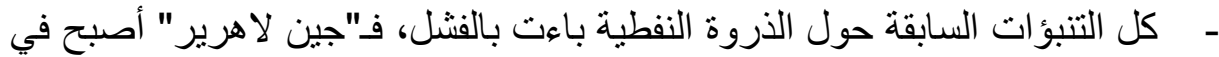

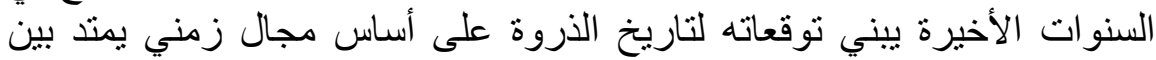

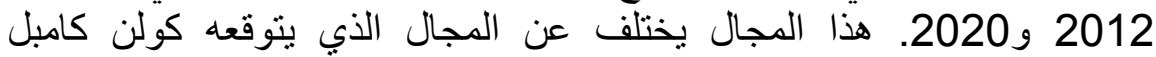
و المحدد بين 2006 و 2010.

- إن القصور الرئيسي لنظرية الذروة النفطية هو اهمالها للمتغيرات غير الجيولوجية التي أدت في السابق ويمكن أن تؤدي في المستقبل الفئل الفي زيادة احتياطات النفطو الغاز الطُبيعي.

\section{-العوامل المؤثرة على المكانة المستقبلية للنفط في الاقتصاد العالمي: III}

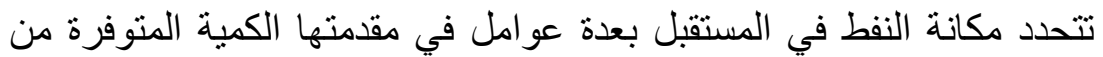
هذا المورد، ثم تأتي القيود المفروضة على على القدرة على الوصول إلى هذئ هذه الموارد، وتتمثل هذا القيود في: ت فئي

لعب التطور التكنولوجي دورا كبير التولي في تطوير الصناعة النفطية في العالم بما التقدم التكنولوجي: -1-III

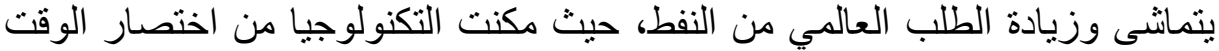

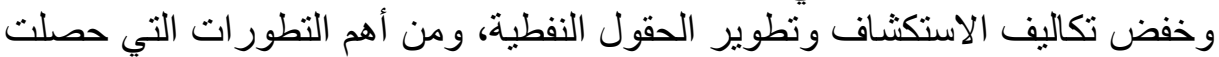

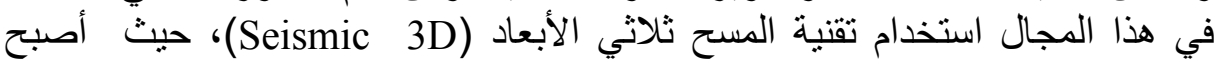


بالإمكان رسم خريطة كاملة عن الطبقات الصخرية و عرضها على شاثشات ضخمة في معامل مخصصة تمكن الجيولوجيين من مطالعتها والتحكم بها والنظة النظر إليها وتحليلها.

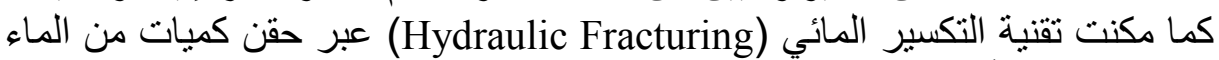

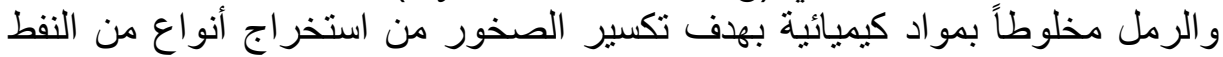

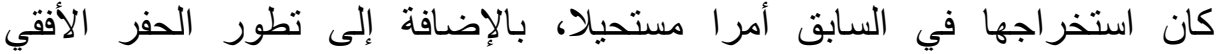
(Horizontal Drilling)

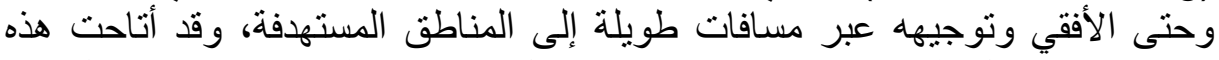

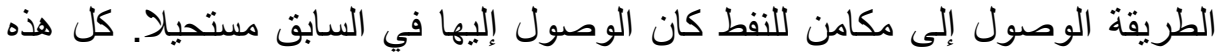

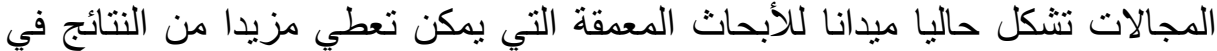

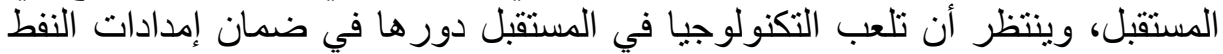
في العالم خاصة من خلال:

I-1-1II

تطورت في السابق نسبة اكتشاف حقول جديدة إلى إجمالي الآبار المستكثفة

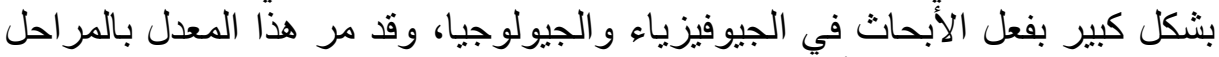

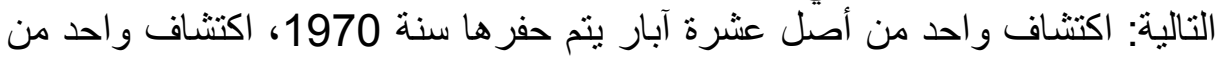

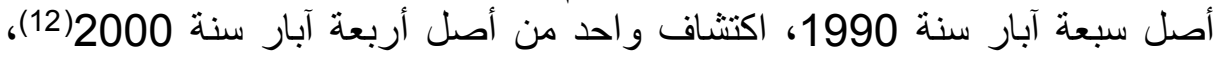

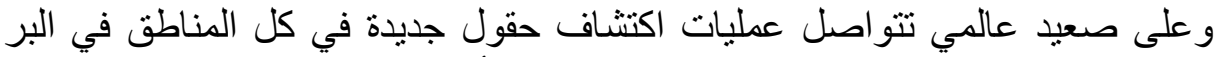

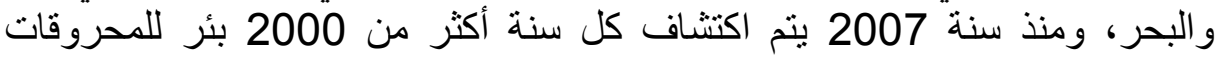

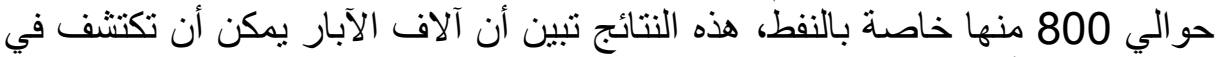

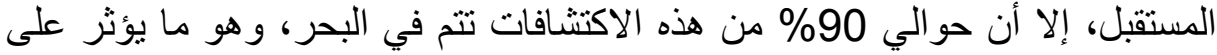

معدل الاستخلاص هول الاصناص: النسبة بين النفط الممكن استخر اجه بالطرق والتقنيات

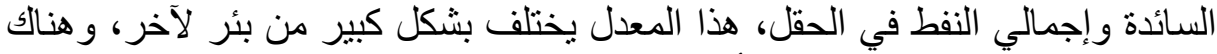

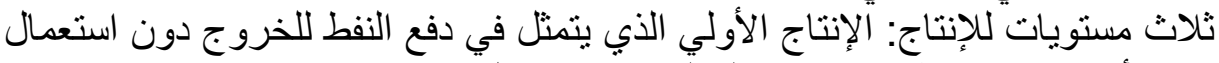

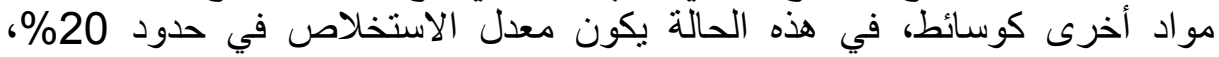

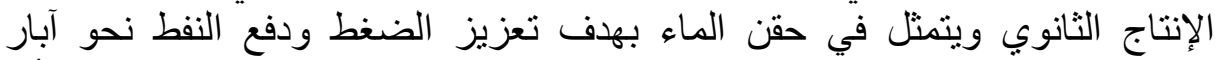

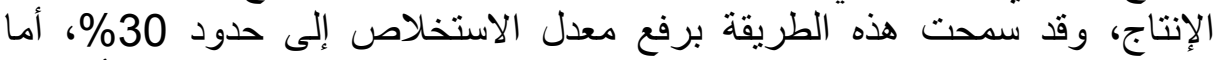

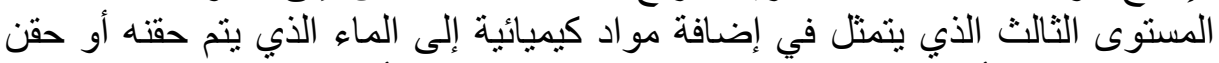

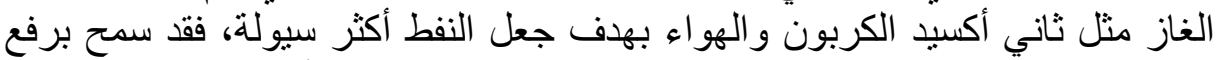

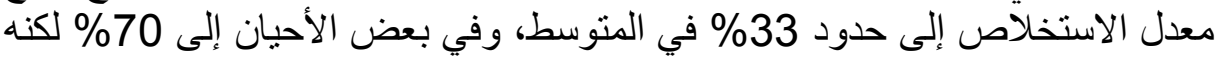

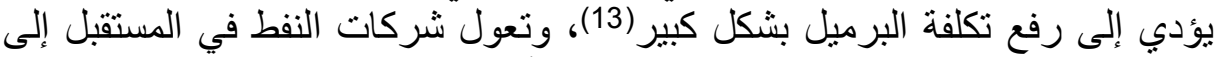

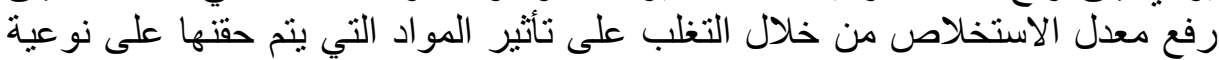
النفط من حيث الكثافة واللزوجة، وتأثير هذه الطريقة على كلفة النفئ النف المستخر ج.

\section{-3-1-III}

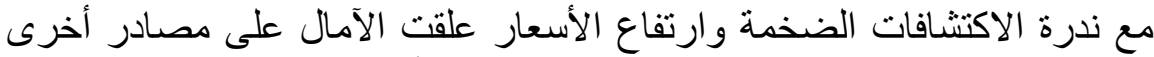

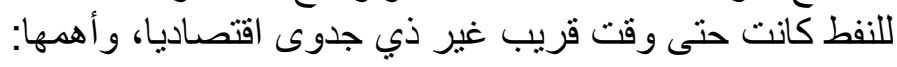

- الرمال النفية (oil sands): وهو نفط يتميز عن النفط الخام بكثافته ولزوجئه

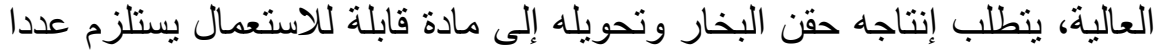

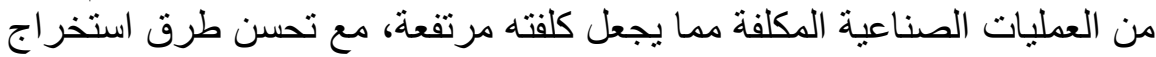

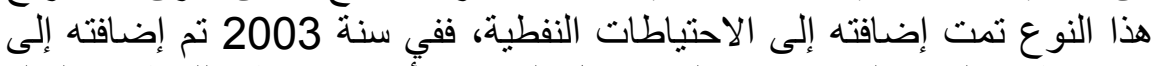

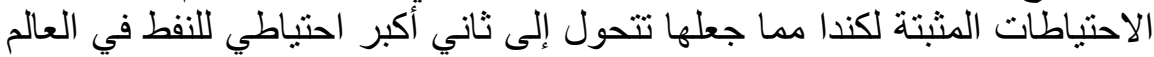




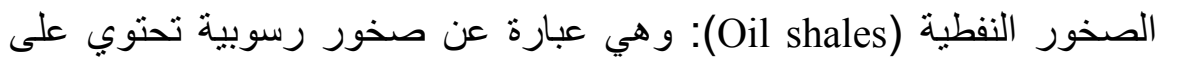

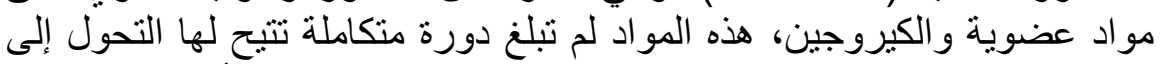

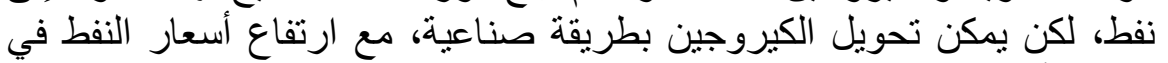

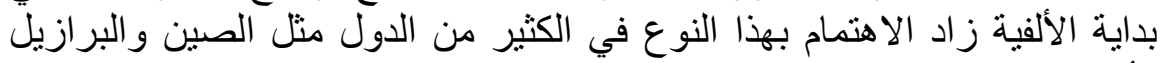
و وألمانيا.

- الغاز الصخري (Shale Gas): وهو غاز محبوس داخل صخور رسوبية تتميز

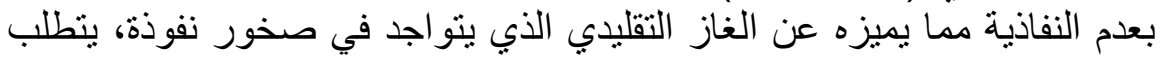

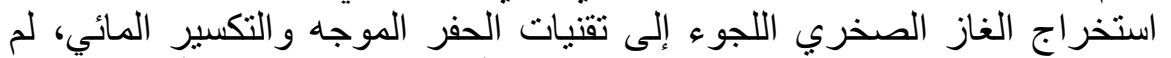

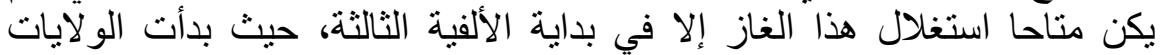

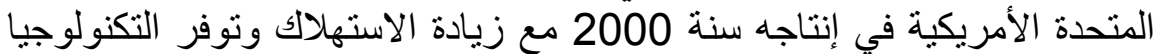

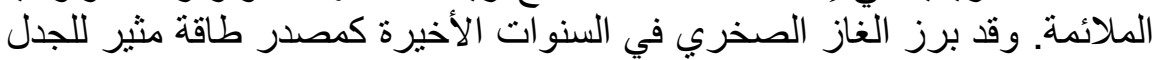

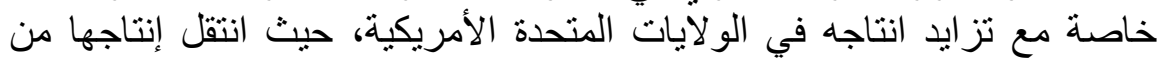

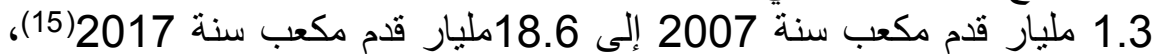

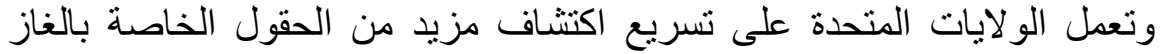

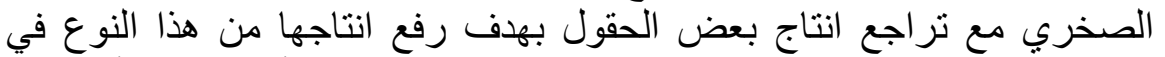

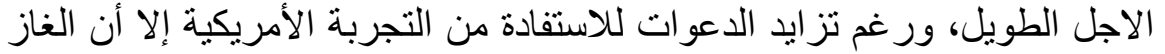

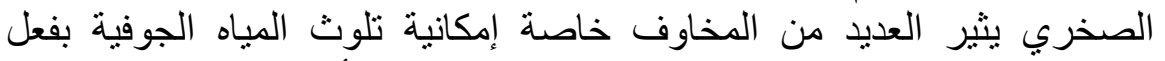

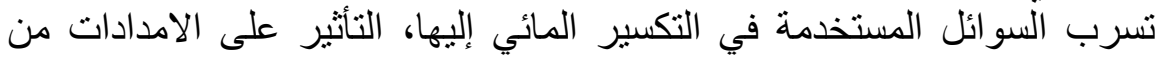

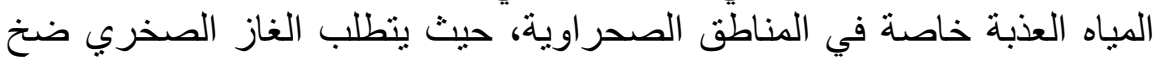

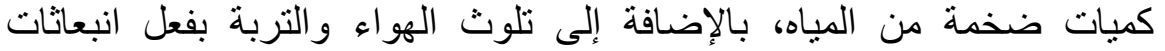

$$
\text { المعدات المستعملة وغاز ات الدياه الإنيئة }
$$

- النفط الصخري (Shale Oil/Tight Oil): وهو لا يختلف عن النفط العادي إلا في

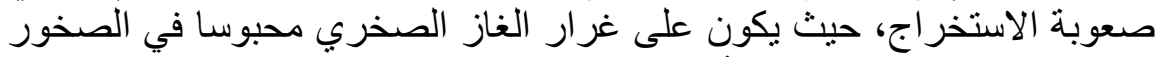
الرسوبية غير النفوذة، ويمكن أن يتو اجد في نفس البئر مع الغاز الصخري الصغي.

-تطور مصادر الطاقة البليلة والتحديات البيئية:

أكدت اللجنة الدولية لتغير المناخ (IPCC) سنة 2005 أن تركيز ثناني أكسيد

الكربون (CO2) في الجو كان ثابتا على مدى الألف سنة الألف الأخيرة في حدود 280

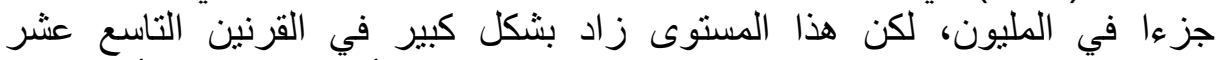

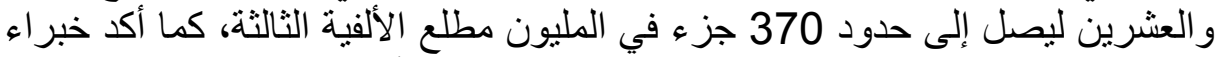

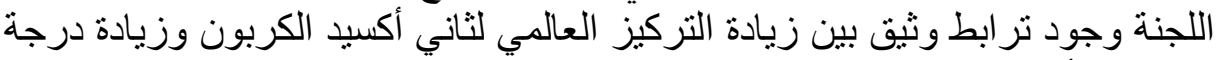

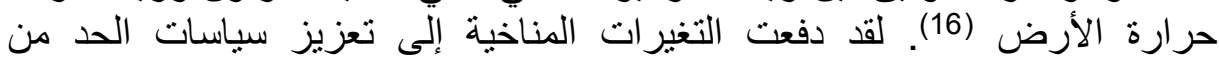

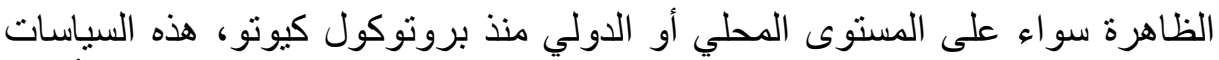

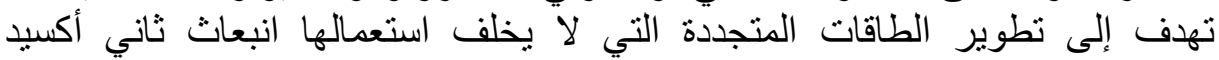

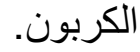

وقد تطورت الاستثمارات في قطاع الطاقات المتجددة على الصعيد العالمي

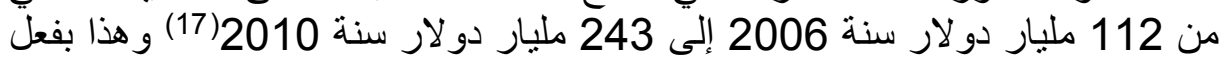

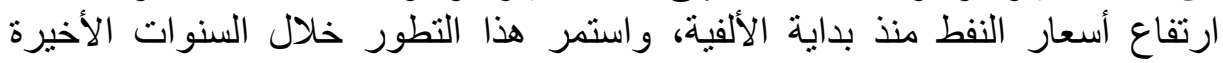

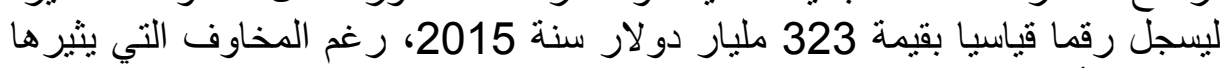

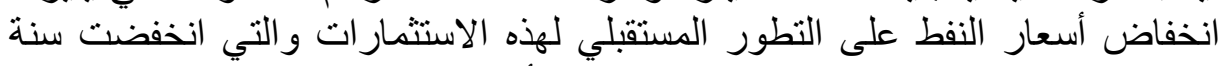

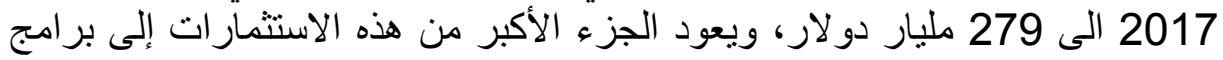

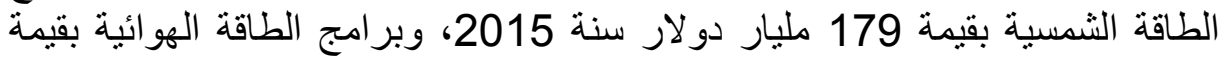
124 مليار دولار خلال السنة نفسها(18)، وتلتوقع الوكالة الدولية للطاقة المتجددة 
(IRENA)

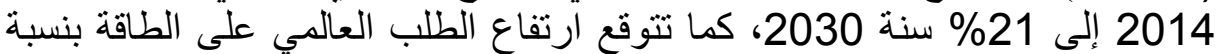

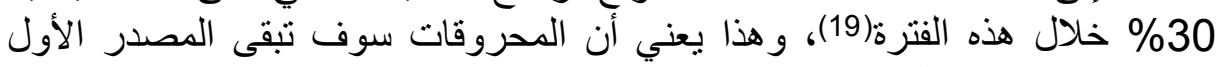

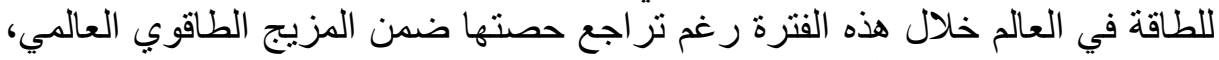

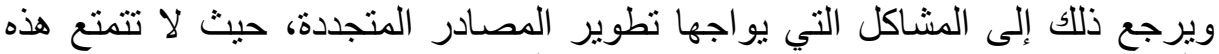

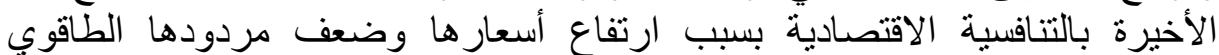

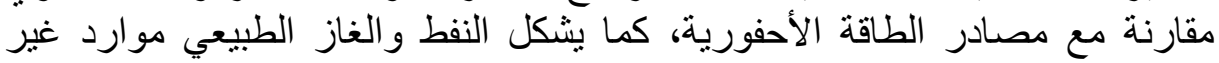

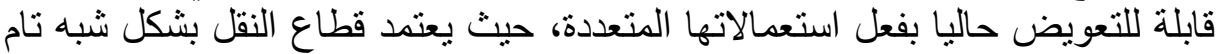

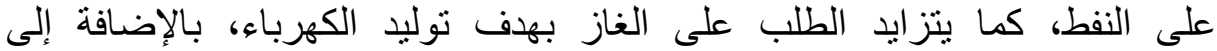
استخدامهما كمو اد أولية في الصناعة البنروكيماوية .

المتروثة-3II

تعمل الأسعار على التوفيق بين العرض والطلب على المصادر المختلفة المقارة

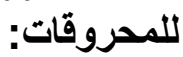

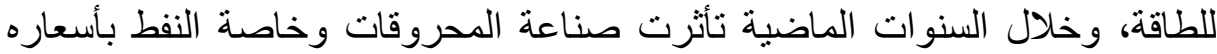

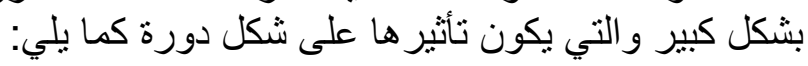

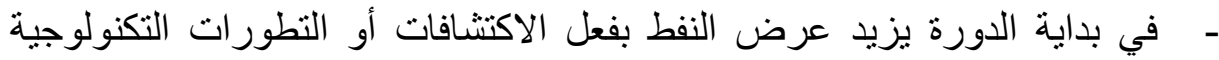
ويدفع لانخفاض سعره مما يؤدي إلى زيادة الاستهلاك.

- تيؤدي الأسعار المنخفضة إلى تراجع استثمارات الثركات العاملة في الصناعة

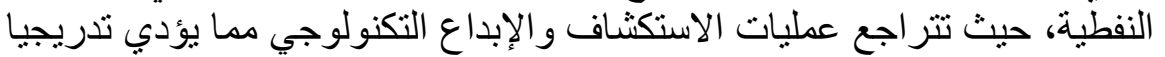

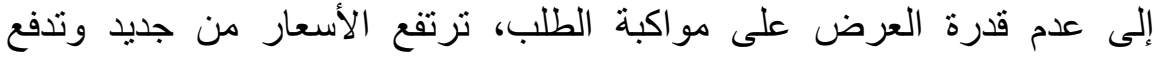

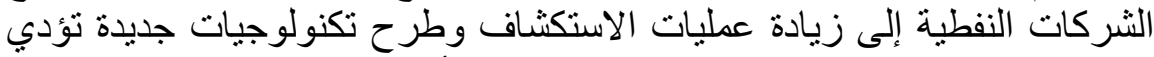

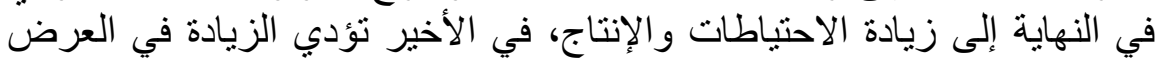

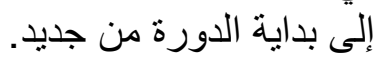

لقد أدت أسعار النفط المنخفضة منذ التسعينات إلى تراجع الاستثمارات في

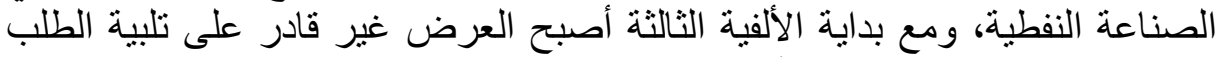

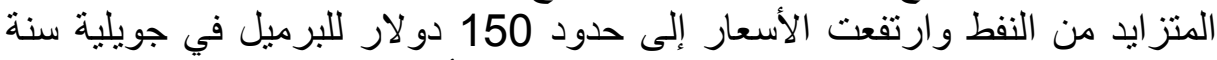

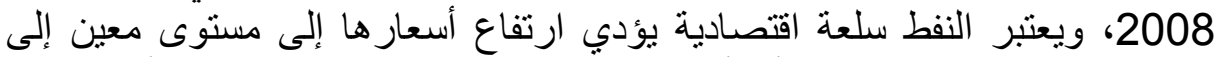

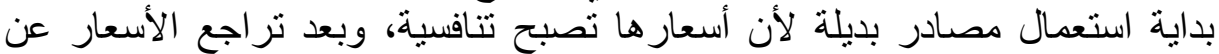

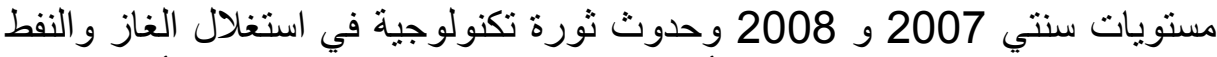

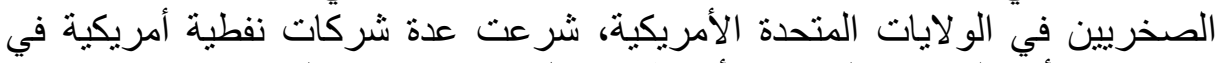

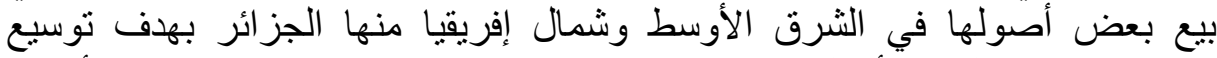

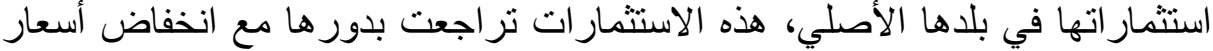

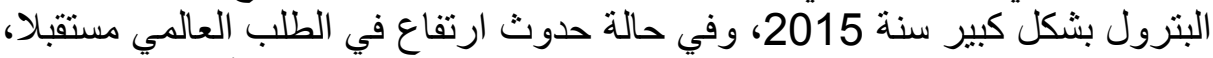
قد لا يمكن للاستثمار ات المنجزة مو اكبة هذا الطلب وبالثالي آرتفاع الكاع الأسعار من جديد. المكانة المستقبلية للمحروقات في الجزائر:-IV

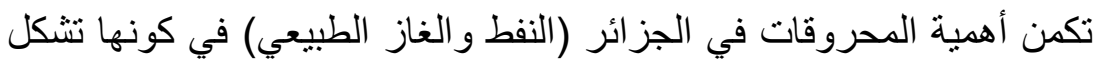

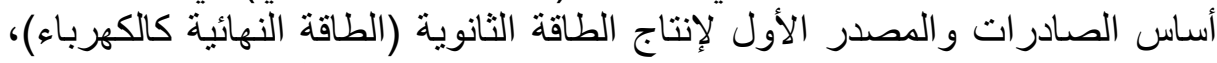
وترتبط مكانتها المستقبلية ارتباطا وثثقا بمكانتها في الاقتصاد العالمي. 
-1-1V

بلغ إنتاج الجزائر من الطاقة الأولية سنة 2017 حوالي 166 مليون طن الأب التئ

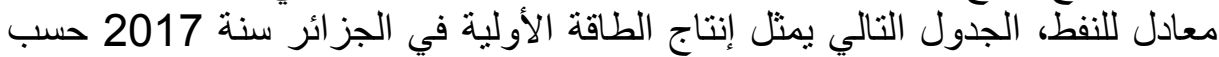

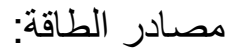

الجدول رقم (02): إنتاج الطاقة الأولية في الجزائر حسب كل مصدر سنة 2017 (الوحدة: ألف طن معادل للنفط).

\begin{tabular}{|c|c|c|}
\hline النسبة المئوية & الكمية & \\
\hline 32.9 & 54564 & نفط خام \\
\hline 6.3 & 10436 & مكثفات \\
\hline 55 & 91286 & غاز طبيعي \\
\hline 5.7 & 9416 & غاز البترول المميع \\
\hline 0.1 & 150 & كهرباء أولية \\
\hline 0.00 & 10 & محروقات صلبة \\
\hline 100 & 165863 & المجموع \\
\hline
\end{tabular}

Ministère de l'énergie et des mines, bilan énergétique national de l'année 2017, édition 2018.

من الجدول نلاحظ الهيمنة التامة للمحروقات على حصيلة الطاقة الأولية المنتجة، حيث تشكل المصدر الأول بحو الي 99.9\%، أما الطاقات المتجددة (الهوائية والشمسية) فهي لا تزال هامشية. لقد شهد إنتاج الطاقة الأولية في السنوات النية الأخيرة

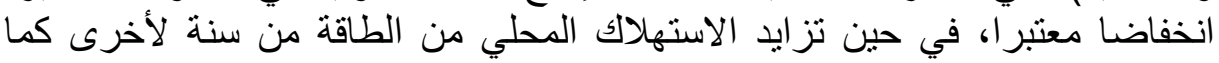

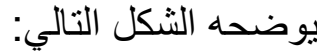

الثكل رقم (04): تطور إنتاج واستهلاك الطاقة في الجزائر منذ سنة 2000

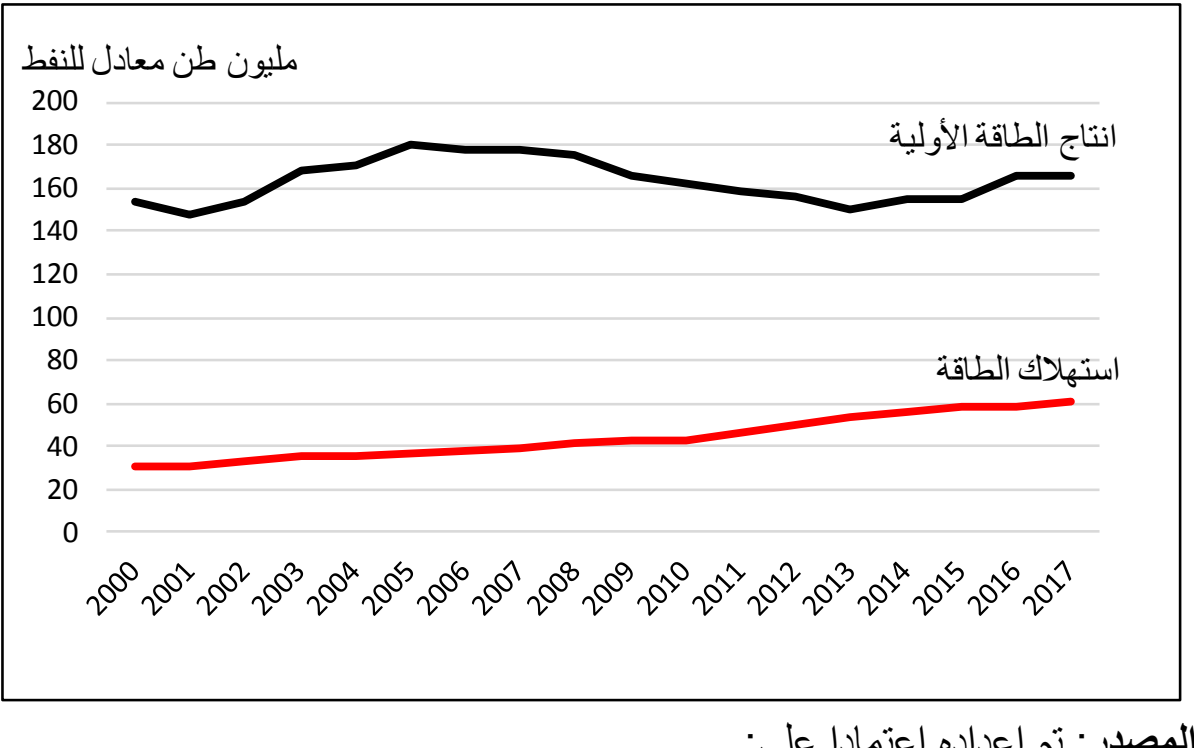

Ministère de l'énergie et des mines, Bilan énergétique national, éditions 2001- 2018.

ففي سنة 2005 بلغ الاستهلاك المحلي من الطاقة 36 مليون طن معادل

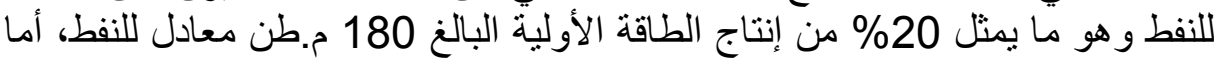

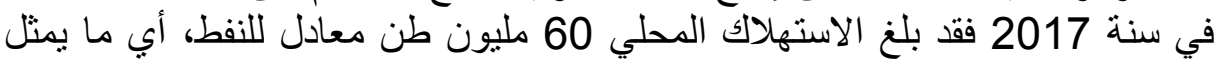


36 من مجموع إنتاج الطاقة الأولية البالغ 166 مليون طن معادل للنفط خلال

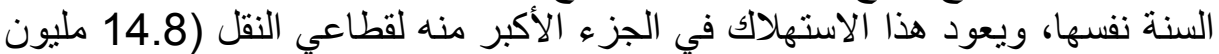

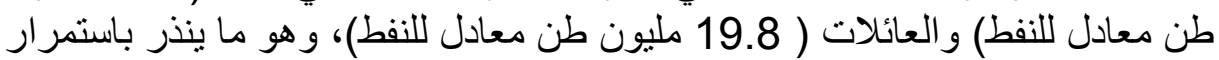

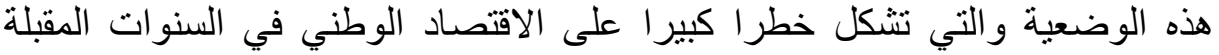

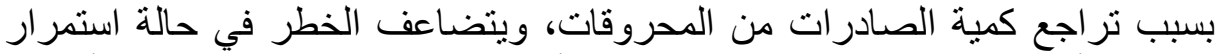

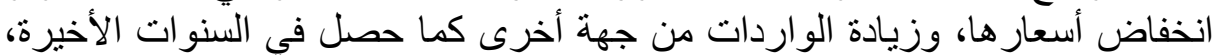

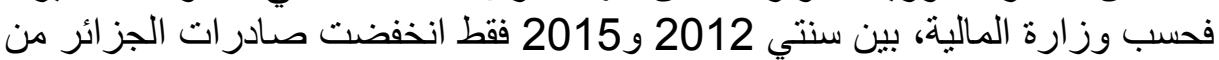

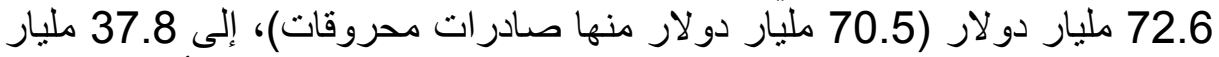

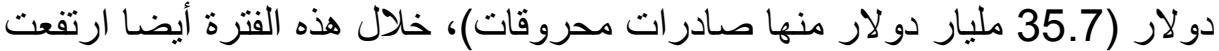

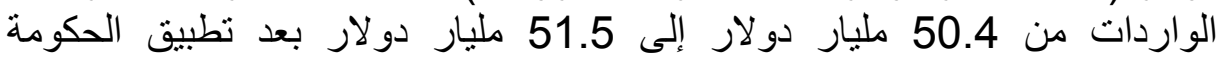
لإجراءات التقتثف، حيث وصلت الواردات التات قبل ذللك سنة 2014 إلى 58.6 مليار

\section{L-داسة الذروة النفية في الجزائر:}

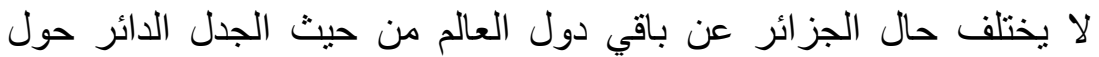

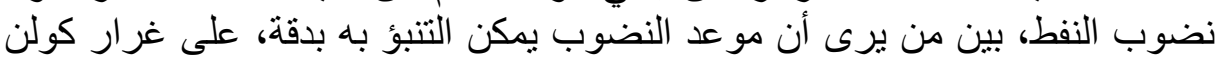

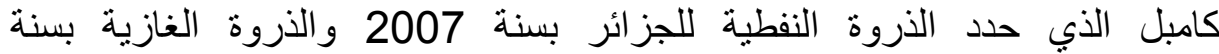

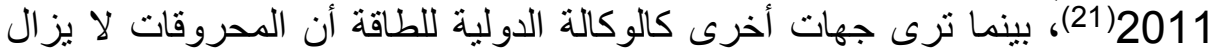

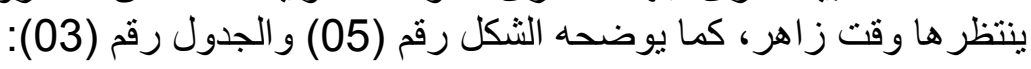

الثكل رقم (05): التوقعات المستقبلية لإتتاج النفط (كل السوائل) والغاز الطبيعي في الجزائر حسب كولن كامبل النيل

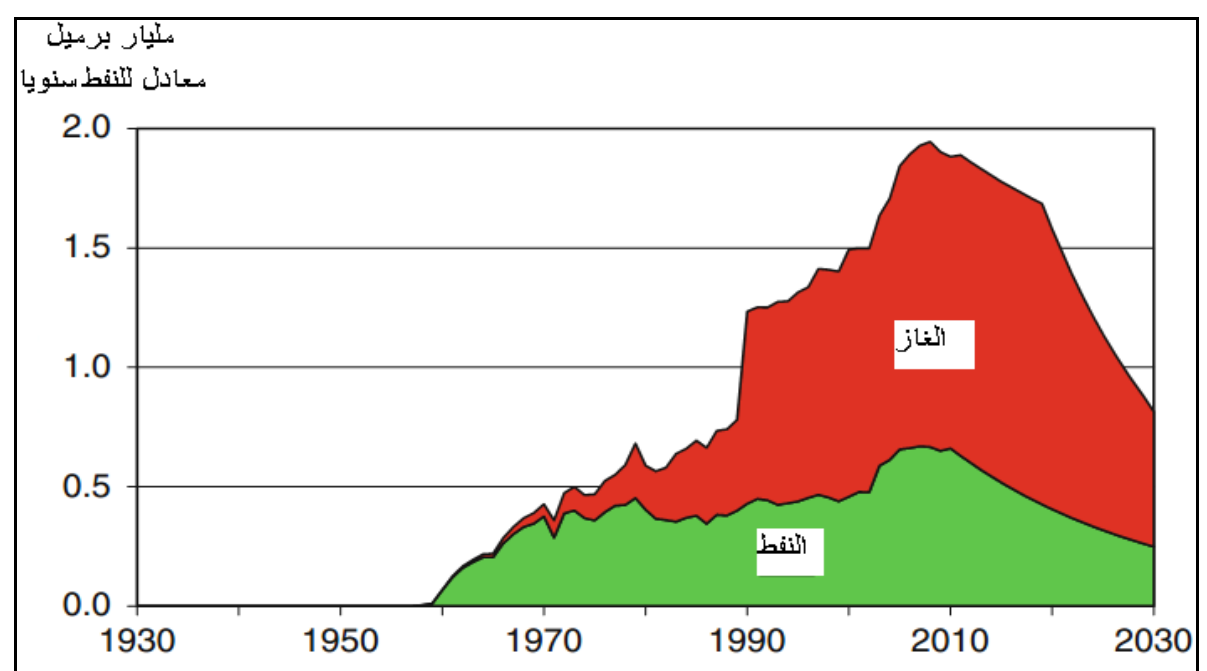

Source: Colin Campbell, Campbell's Atlas of Oil and Gas Depletion, P.23.

من المنحنى نلاحظ أن الإنتاج السنوي للنفط في الجزائر بلغ سنة 2007 فئل

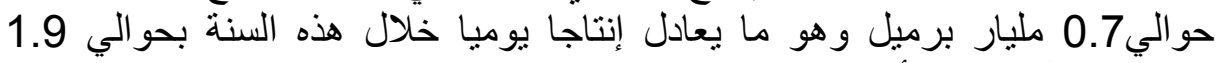

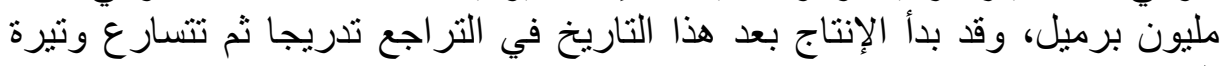

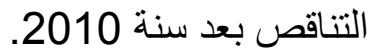

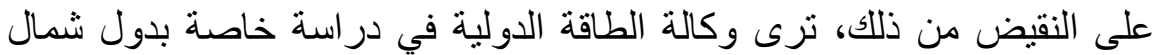

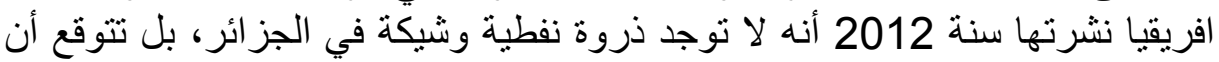
يزيد إنتاج النفط الجزائري في المستقبل بشكل طفيف ليستقر في حدود 2012 مليون برميل فئل 
يوميا سنة 2035، أما بالنسبة للغاز الطبيعي فتتوقع زيادة في الإنتاج تصل إلى حدود

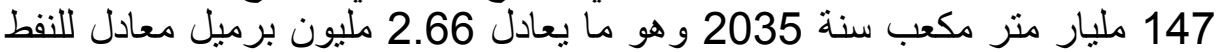

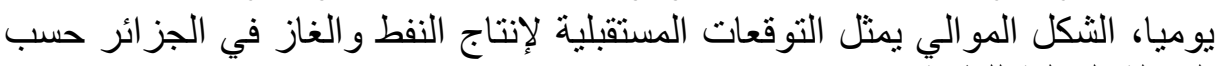

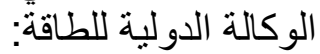

الجدول رقم (03): التوقعات المستقبلية لإنتاج النفط في الجزائر حسب السيناريو المرجعي

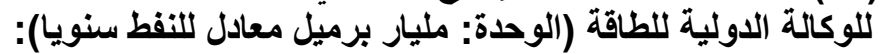

\begin{tabular}{|c|c|c|c|c|c|c|c|}
\hline 2035 & 2030 & 2025 & 2020 & 2015 & 2011 & 1990 & السنو ات \\
\hline 0.73 & 0.73 & 0.693 & 0.693 & 0.693 & 0.657 & 0.474 & إنتاج النفط \\
\hline 0.970 & 0.924 & 0.812 & 0.693 & 0.548 & 0.528 & 0.283 & إنتاج الغاز \\
\hline
\end{tabular}

Source: IEA World Energy Outlook 2012, IEA publications, Paris, P115.

ومماز زاد من مخاوف وصول الجزائر إلى مرحلة الذروة النفطية في السنوات

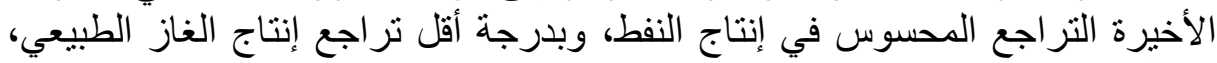
الثكل التالي يوضح تطور الإنتاج السنوي من النفط والغاز منذ سنة 2000

الشكل رقم (06): تطور الإنتاج السنوي للنفط والغاز الطبيعي في الجزائر منذ سنة 2000:

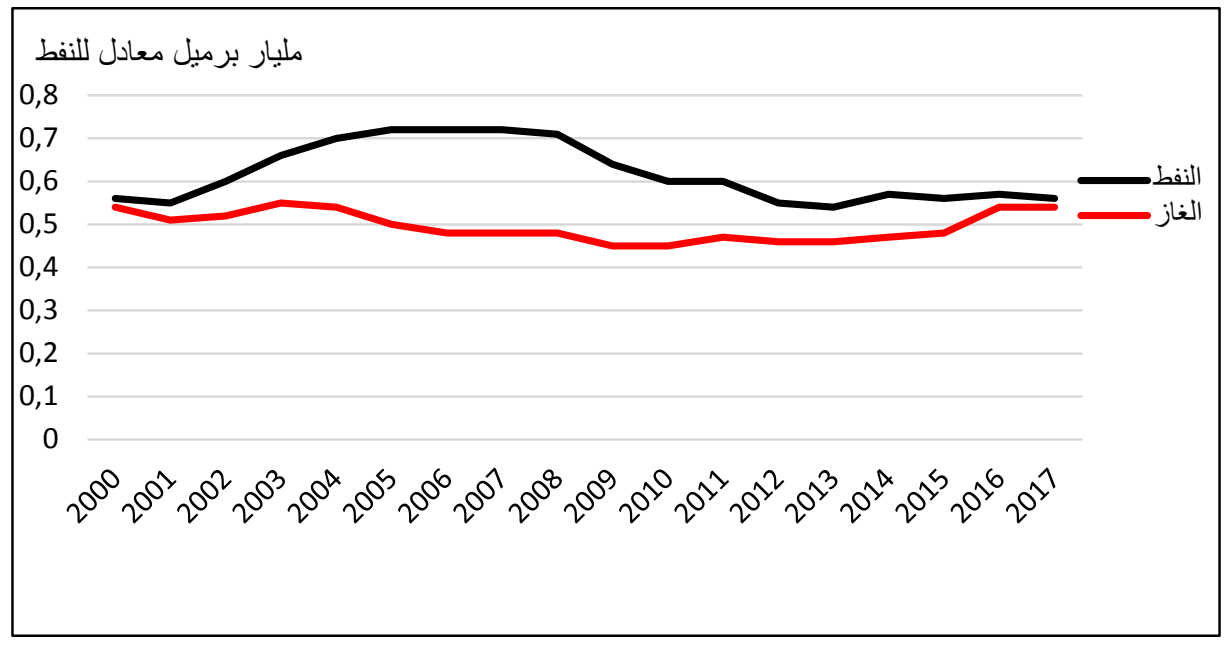

المصدر: تم إعداده اعتمادا على:

British petroleum, BP statistical review of world energy, June 2018.

لكن، هل يعود تراجع إنتاج المحروقات في الجزائر فعلا إلى تخطيها مرحلة

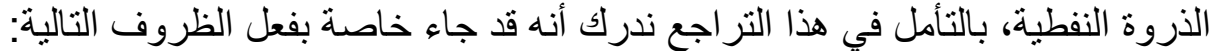

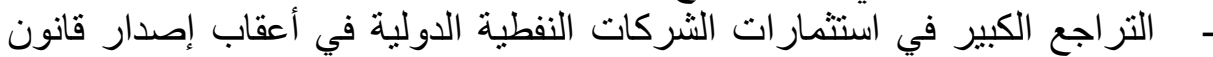

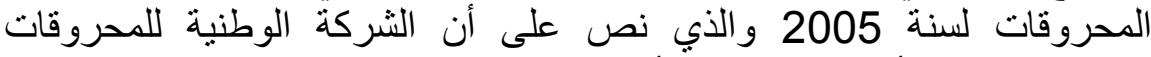

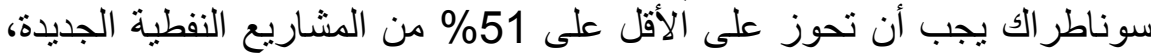

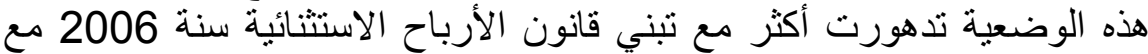

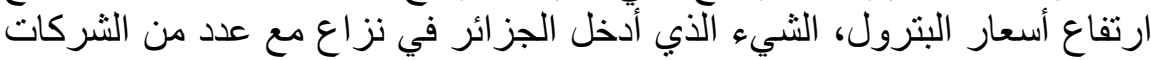

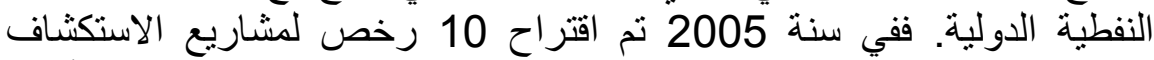
منحت منها 09 رخص، لكن سنة 2008 لم يتم إبرام سوى 4 عقود من أصل الصل 


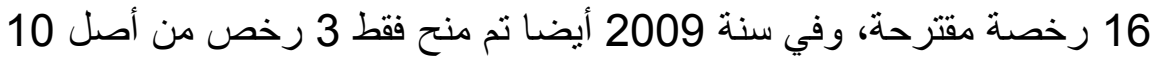
رخص مقترحة (22)، وقد تر اجعت استتمار ات هذه الثركات من 3.3 مليار دو لار

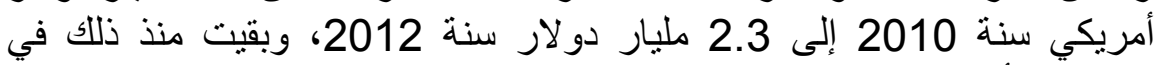
مستويات أقل من حجمها سنة 2010، 2010، حتى وصلت اللى 1.4 مليار دولار سنة (23)2017

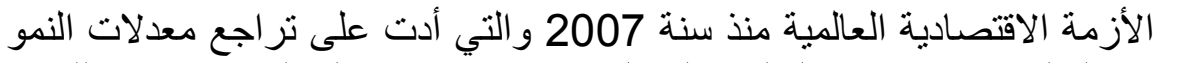

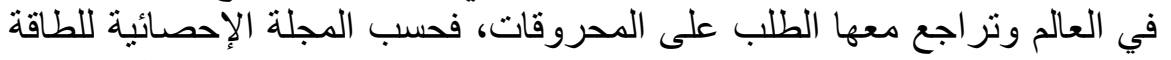

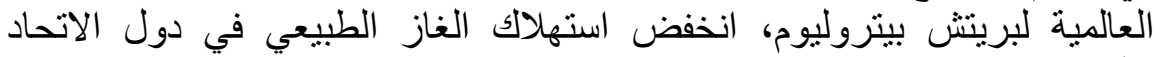

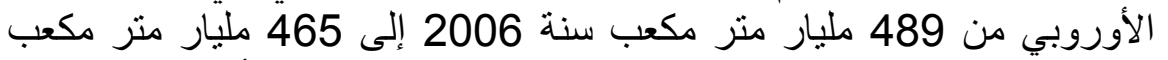

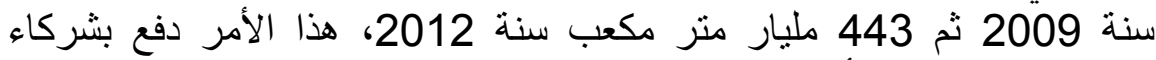

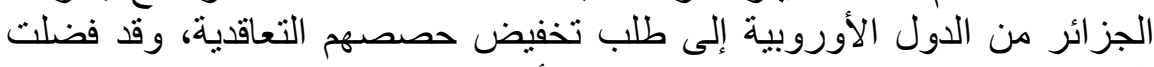

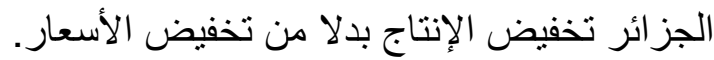

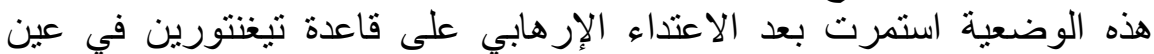

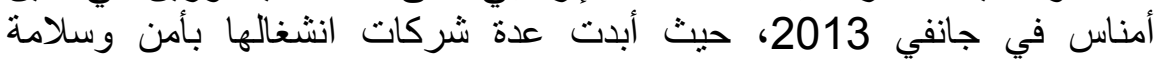

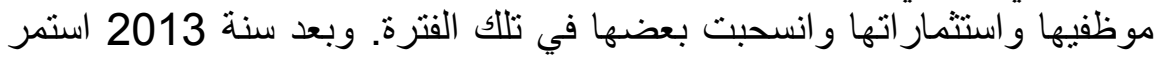

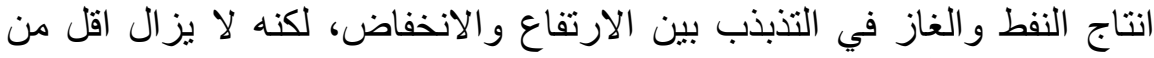

مستوى الإنتاج لسنة 2008 النئة

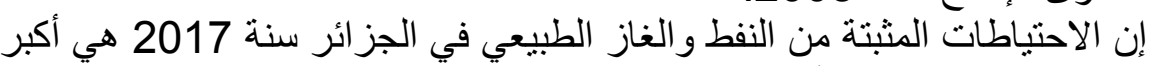

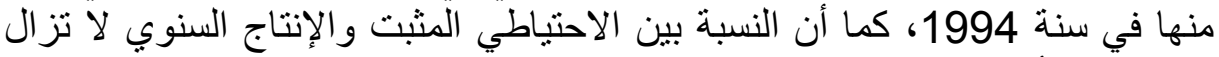
مستقرة منذ أكثر من عقدين بالنسبة للنفط كما يبينه الجدول التالي: التئ التيا

الجدول رقم (04): الاحتياطات المثبتة والإنتاج السنوي من النفط والغاز الطبيعي في

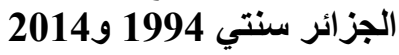

\begin{tabular}{|c|c|c|c|}
\hline 2017 & 1994 & & 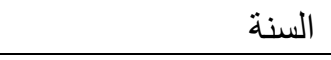 \\
\hline 12.2 & 10 & النفط (مليار برميل) & \multirow{2}{*}{ الاحتياطات المثبتة (R) } \\
\hline 4300 & 3000 & الغاز الطبيعي )مليار م³) & \\
\hline 562 & 483 & النفط (مليون برميل) & \multirow{2}{*}{ الإنتاج السنوي (P) } \\
\hline 91 & 54 & الغاز الطبيعي (مليار منز مكعب) & \\
\hline 22 & 21 & النفط & \multirow[t]{2}{*}{ R/P (بالسنوات) R } \\
\hline 47 & 56 & الغاز الطبيعي & \\
\hline
\end{tabular}

British petroleum, BP statistical review of world energy, June 2015 and June 2018.

التحديات التي تواجه إنتاج المحروقات في الجزائر:-3-IV

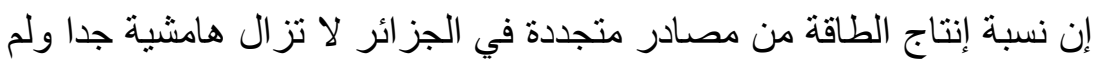

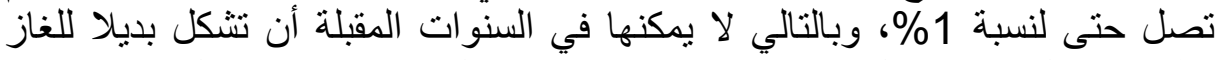

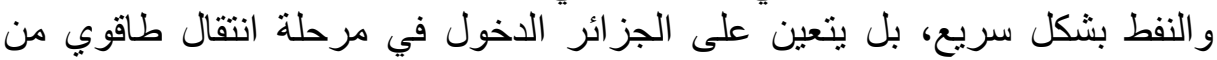

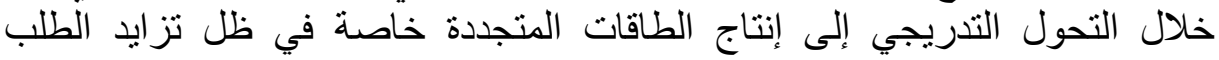
المحلي، خلال هذه المرحلة يواجه إنتاج المحروقات التحات التحديات التالية:

\section{1-3-IV}

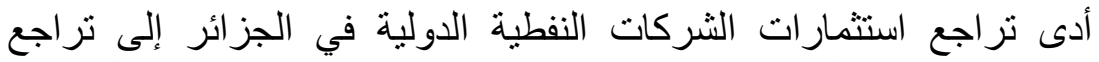

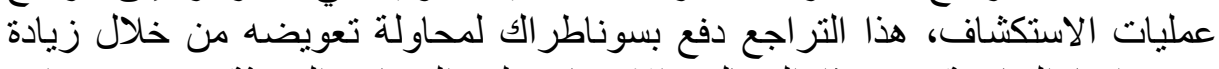

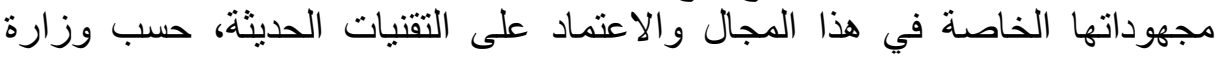


الطاقة والمناجم، مست عمليات المسح ثنائي الأبعاد 8696 كم2 سنة 2011 مقابل 16778 كم2 سنة 2011، ما يمثل تر اجعاً بنسبة 48\%؛ الابعاد، في المقابل زادت عمليات

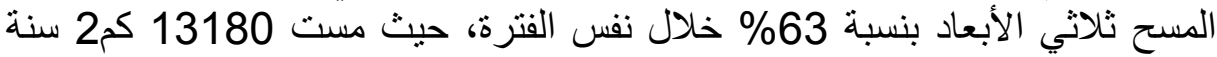
2012 مقابل 8097 كم2 سنة 2012، واستمر الارتفاع ليسجل رقما قياسيا سنة 2015 ليمس حوالي 2017 كابل 18415 كم2، مع تسجيل انخفاض طفيف سنوات 2016

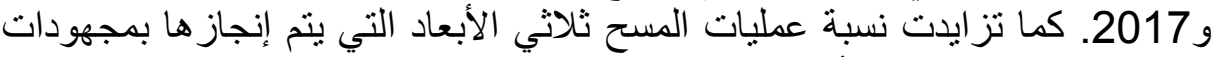
سوناطر الك فقط من سنة لأخرى، بحيث انتقلت هذه النسبة من 37 37

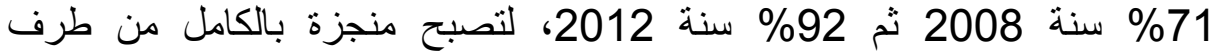
سوناطر الك سنة 2017. أما عمليات الحفر فقد مست 258 كم سنة 2012 أنجزت

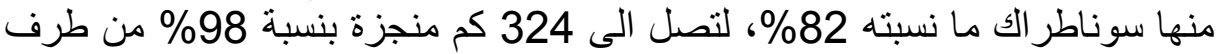

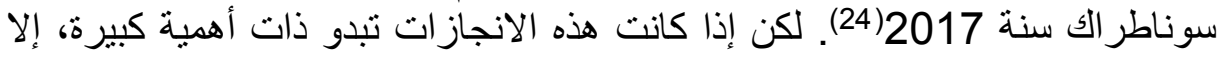
أنها نثير من جهة أخرى إلى أن الثركات النفطية الدولية لم تعد تقوم بعمليات

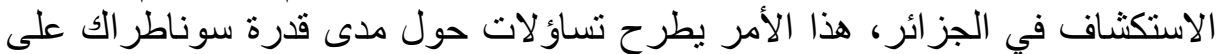
مو اجهة تحديات المرحلة المقبلة.

-2-3-IV تتوفر الجزائر على حقول قديمة لكنها ذات أهية أهمية كبيرة من حيث حجم

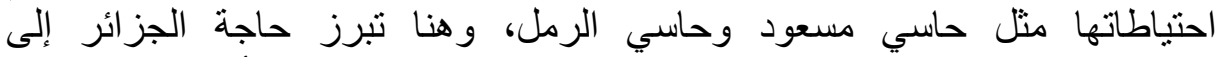

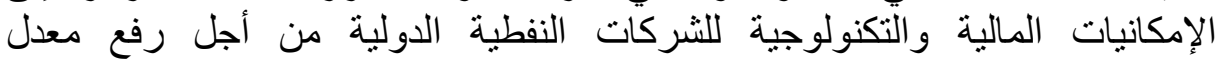
الاستخلاص في هذه الحقول وضمان الزيادة في الإنتاج.

3-3-IV تشير التقديرات الأولية لإدارة معلومات الطاقية الطاتئة الأمريكية وهي هيئة تابعة

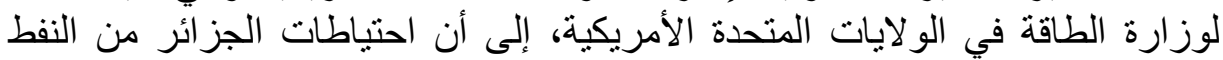

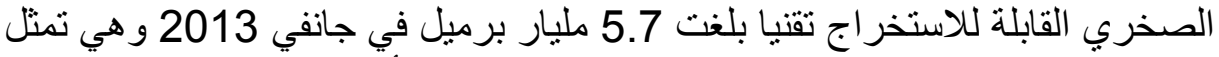

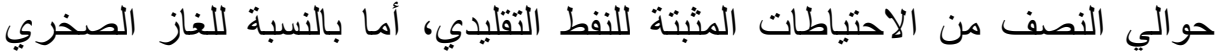

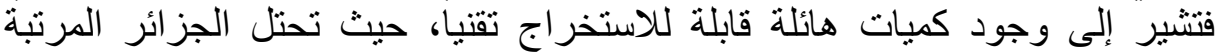

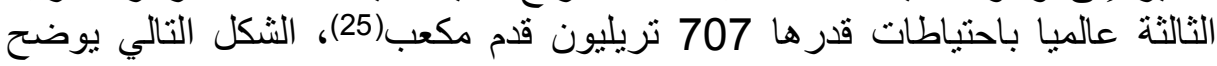

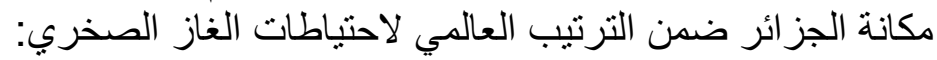

الجدول رقم (05): يبين أكبر احتياطات الغاز الصخري في العالم في بداية سنة 2013

\begin{tabular}{|c|c|c|}
\hline 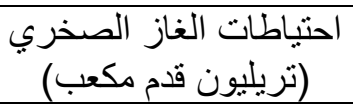 & 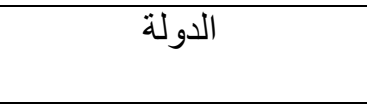 & الترتيب العالمي \\
\hline 1115 & الصين & 1 \\
\hline 802 & الأرجنتين & 2 \\
\hline 707 & الجز ائر & 3 \\
\hline 665 & الو لايات المتحدة الأمريكية & 4 \\
\hline 573 & كندا & 5 \\
\hline 545 & المكسيك & 6 \\
\hline 437 & أستر اليا & 7 \\
\hline 390 & جنوب إفريقيا & 8 \\
\hline 285 & روسيا & 9 \\
\hline 245 & البرازيل & 10 \\
\hline 7299 & 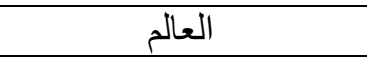 & \\
\hline
\end{tabular}

Source: US Energy Information Administrations:

http://www.eia.gov/analysis/studies/worldshalegas/pdf/fullreport.pdf, $(12 / 11 / 2017)$. 
ويدفع تزايد الاستهلاك المحلي من الطاقة بالإضافة اعتماد الاقتصاد الوطني

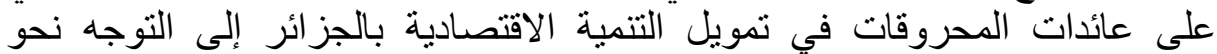

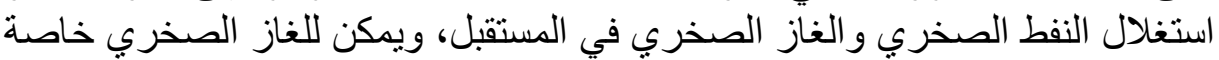
أن يضمن للجزائر فترة للانتقال الطاقوي تمتد لسنوات، فالجزائر أكبر منتج للغناز الفاز

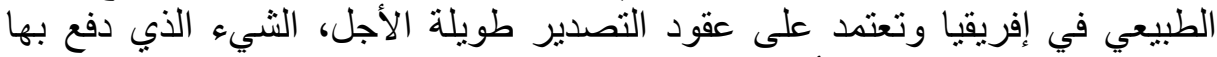

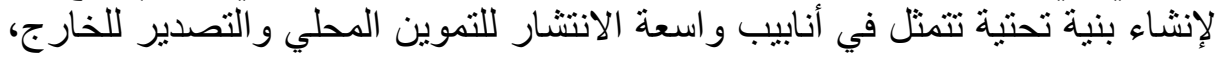

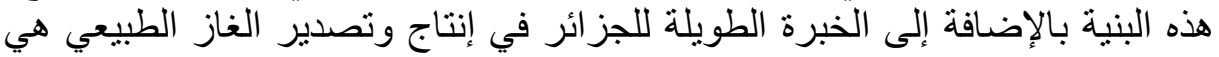

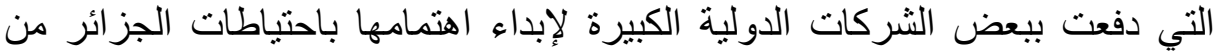

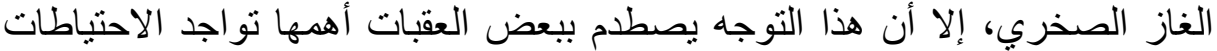

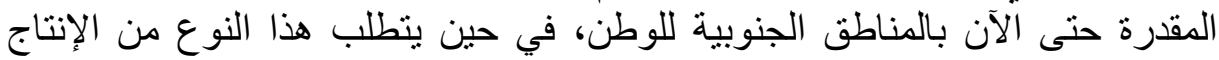

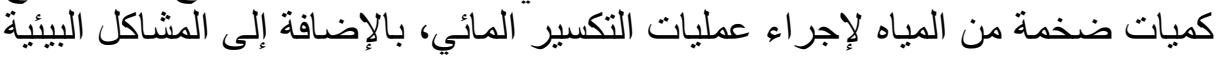

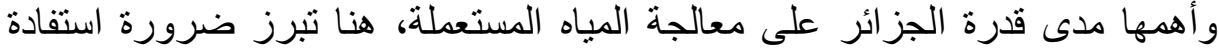

الجزائر من تجارب الدول الر ائدة في هذا المجال خاصة الو لائات المتحدة الأمريكية.

\section{نتائج الاراسة:}

من خلال هذه الدراسة توصلنا إلى النتائج التالية، والتي تؤكد صحة فرضيات

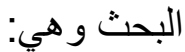

- لا يمكن الزعم بأن العالم قد وصل أو تعدى مرحلة الذروة النفطية والغازية لأن

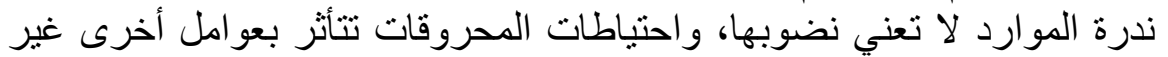

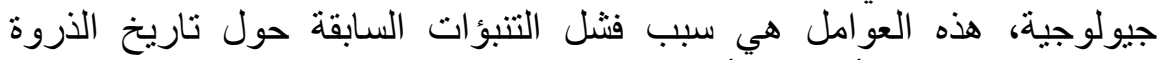
النفطية، و التي يمكن أن تفشل أيضا في المستقبل.

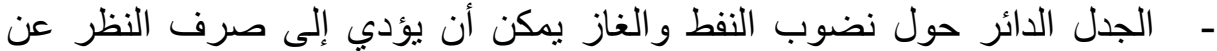

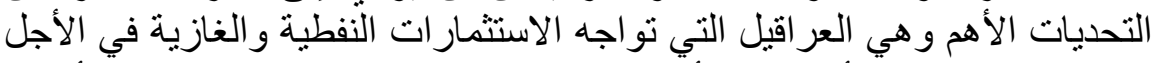

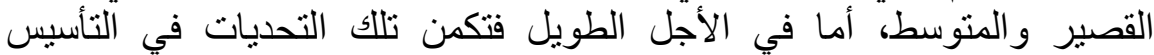
لمرحلة انتقال نحو استعمال الطاقات المتجددة.

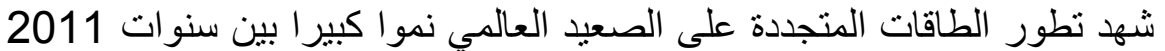

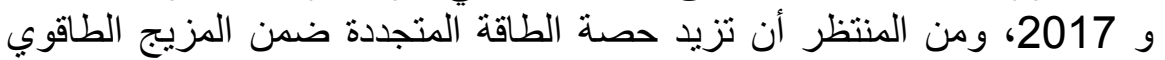

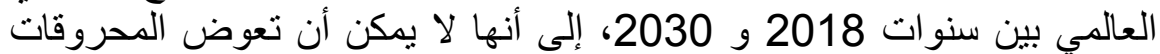

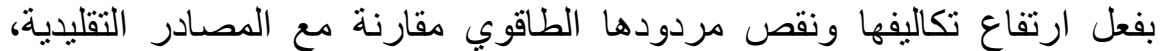

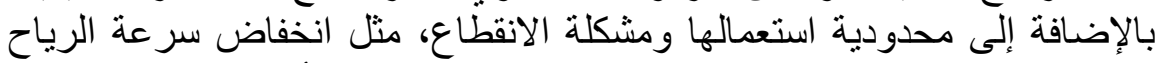

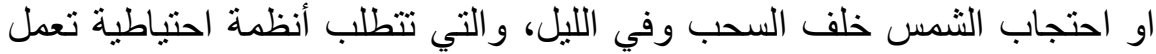
على الوقود الاحفوري لضمان استمر ارية التزود بالطاقة عند الانقطاع، بالإضافة

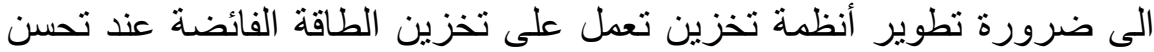

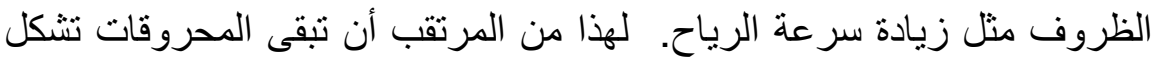

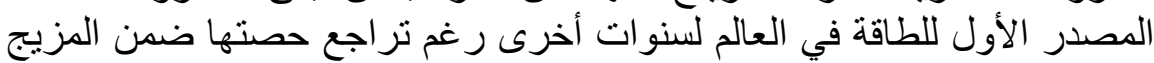
الطاقوي العالمي، بسبب تزايد الطلب على النفط خاصة في قطاع النقل نظر الترا

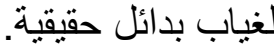
يرتبط مستقبل المحروقات في الجزائر بما يجري من تطورات بلى على الصعيد العالمي، وتتطلب تحديات المرحلة المقبلة وجود بيئة تشريعية ملائمة ومستقرة بهدف نتجيع استثمارات الثركات النفطية الدولية والاستفادة من إمكاناتها

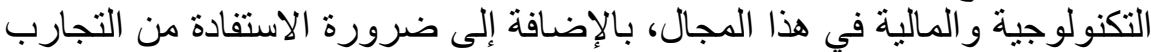

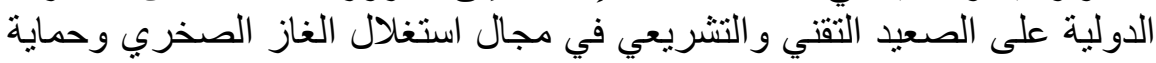

- تطوير قطاع المحروقات وزيادة إنتاجها في الجزائر يجب أن يكون في إطار

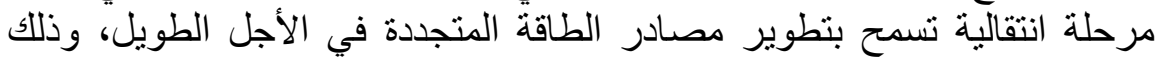


مسايرة للتطورات العالمية في هذا المجال والتي تؤدي إلى تحول الاستهلاك

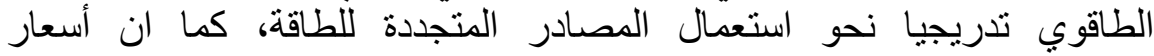

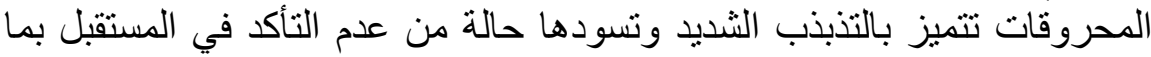
ير هن مستقبل الاقتصاد الوطني الذي يعتمد حاليا على الصادرات من الصن المحروفات.

عند دراسة مستقبل المحروقات يخلط أنصار نظرية الذروة النفطية والغازية

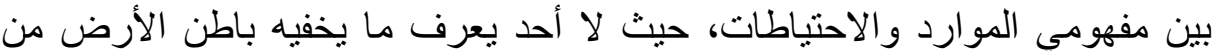
موارد، و التي يمكن أن تضاف إلى الاحتياطات مع التقدم التقني، إن مكانة النفط و الغاز التان

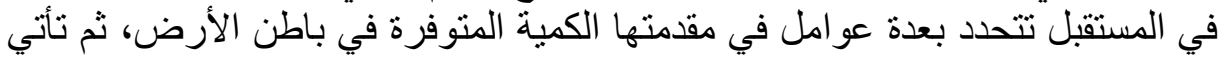

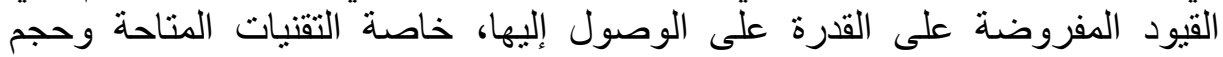

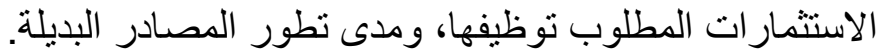

(1) Gilbert Naudet et Paul Reuss (2008), énergie-électricité et nucléaire, EDP Sciences, Paris, P.39-40.

(2) Mohamed Benhaddadi et Guy Olivier (2008), dilemmes énergétiques, Presses de l'Université du Québec, Québec, P.08.

(3) Gilbert Naudet et Paul Reuss, énergie-électricité et nucléaire, op.cit, P.43.

(4) - International Energy Agency (2018), world energy outlook 2018, IEA Publications, Paris.

(5) - Kjell Aleklett and Michael Lardelli (2012), Peeking at Peak oil, Springer, London, P.101.

(6) - Colin J. Campbell and Jean H. Laherrère (1998), the End of Cheap Oil, Scientific American review, 278(03), USA, pp.78-83.

Visité : 20/11/2016, www.oilcrisis.com/campbell/endofcheapoil.pdf

(7) - Kjell Aleklett and Michael Lardelli, Peeking at Peak oil, op.cit, P.117.

(8) - Colin Campbell (2005), the assessment and importance of oil depletion, in "the final energy crisis", edited by Andrew McKillop, Plutobooks, London, P.37.

(9) - Richard Heinberg (2005), The Party's Over, New Society Publishers, second edition, Canada, P.143-444.

(10) - Leonardo Maugeri (2006), the age of oil-the mythology, history, and future of the world's most controversial resource, PRAEGER, London, P.189.

(11) - Duncan Clarke (2007), the battle for barrels-peak oil myths and world oil futures, profile books, London, P35.

(12) - Jean-Louis Bobin et autres (2005), l'énergie de demain, EDP sciences, Paris, P.158.

(13) -Yves Mathieu (2011), le dernier siècle du pétrole- la vérité sur les réserves mondiale, éditions TECHNIP, Paris, P.23.

(14) - Steven M. Gorelick (2010), Oil Panic and the Global Crisis, Blackwell publishing, Oxford, P.169.

(15) - US Shale Production,visité: 25/11/2016 https://www.eia.gov/dnav/ng/hist/res_epg0_r5302_nus_bcfa.htm

(16) - روبرت إفانز(2011)، شحن مستقبلنا بالطاقة ـمدخل إلى الطاقة المستدامة، ترجمة د.فيصل حردان،

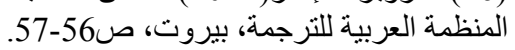

(17) - Renewable Energy Policy Network for the 21st Century, renewables global status report: 
www.ren21.net/wp-content/uploads/2017/06/17-

8399_GSR_2017_Full_Report_0621_Opt.pdf

(18) - Renewable Energy Policy Network for the 21st Century, renewables global status report:

www.ren21.net/wp-content/uploads/2018/06/17-

8652_GSR2018_FullReport web final_pdf

(19) - International Renewable Energy Agency, Roadmap for A Renewable Energy Future, $\quad$ visited: 25/01/2017: www.irena.org/DocumentDownloads/Publications/IRENA_REmap_2016 edition rep ort.pdf

$$
\text { (16) -وزارة المالية الجز ائرية، لمزيد من التفاصيل على الموقع: }
$$

www.mf.gov.dz/article/48/Zoom-sur-les-Chiffres-/142/Balan,ce-commerciale.html, $(20 / 11 / 2017)$

(21) - Colin Campbell (2013), Campbell's Atlas of Oil and Gas Depletion, Springer, London, P.23.

(22) - Ministère de l'énergie et des mines, bilan des réalisations du secteur de l'énergie et des mines 1962-2010, édition 2011, p 16.

(23) - Ministère de l'énergie et des mines, bilan des réalisations du secteur de l'énergie et des mines, éditions 2006-2018.

(24) - Ministère de l'énergie et des mines, bilan des réalisations du secteur de l'énergie et des mines, éditions : 2000-2008, 2012.

(25) - U.S. Energy Information Administration, world shale gas and shale oil resource assessment, visited: 11/12/2017:

http://www.eia.gov/analysis/studies/worldshalegas/pdf/fullreport.pdf 